\title{
Testing matter effects in propagation of atmospheric and long-baseline neutrinos
}

\author{
M.C. Gonzalez-Garcia ${ }^{a, b}$ Michele Maltoni $^{c}$ and Jordi Salvado ${ }^{d}$ \\ ${ }^{a}$ C.N. Yang Institute for Theoretical Physics, \\ State University of New York at Stony Brook, \\ Stony Brook, NY 11794-3840, U.S.A. \\ ${ }^{b}$ Institució Catalana de Recerca i Estudis Avançats (ICREA), \\ Departament d'Estructura $i$ Constituents de la Matèria \\ and Institut de Ciencies del Cosmos, Universitat de Barcelona, \\ Diagonal 647, E-08028 Barcelona, Spain \\ ${ }^{c}$ Instituto de Física Teórica UAM/CSIC, Calle de Nicolás Cabrera 13-15, \\ Universidad Autónoma de Madrid, \\ Cantoblanco, E-28049 Madrid, Spain \\ ${ }^{d}$ Departament d'Estructura $i$ Constituents de la Matèria \\ and Institut de Ciencies del Cosmos, Universitat de Barcelona, \\ Diagonal 647, E-08028 Barcelona, Spain \\ E-mail: concha@insti.physics.sunysb.edu, michele.maltoni@csic.es, \\ jsalvado@ecm.ub.es
}

ABSTRACT: We quantify our current knowledge of the size and flavor structure of the matter effects in the evolution of atmospheric and long-baseline neutrinos based solely on the analysis of the corresponding neutrino data. To this aim we generalize the matter potential of the Standard Model by rescaling its strength, rotating it away from the ee sector, and rephasing it with respect to the vacuum term. This phenomenological parametrization can be easily translated in terms of non-standard neutrino interactions in matter. We show that in the most general case, the strength of the potential cannot be determined solely by atmospheric and long-baseline data. However its flavor composition is very much constrained and the present determination of the neutrino masses and mixing is robust under its presence. We also present an update of the constraints arising from this analysis in the particular case in which no potential is present in the $e \mu$ and $e \tau$ sectors. Finally we quantify to what degree in this scenario it is possible to alleviate the tension between the oscillation results for neutrinos and antineutrinos in the MINOS experiment and show the relevance of the high energy part of the spectrum measured at MINOS.

Keywords: Solar and Atmospheric Neutrinos, Neutrino Physics, Beyond Standard Model ARXIV EPRINT: 1103.4365 


\section{Contents}

1 Introduction 1

2 Formalism 3

3 Results 6

$4 \quad$ No matter effects in $e \mu$ and $e \tau$ sectors $\quad 12$

$\begin{array}{llr}5 & \text { Summary } & 17\end{array}$

$\begin{array}{ll}\text { A Parametrization of the matter potential } & 18\end{array}$

$\begin{array}{ll}\text { B Symmetries and parameter ranges } & 20\end{array}$

\section{Introduction}

It is now an established fact that neutrinos are massive and leptonic flavors are not symmetries of Nature $[1,2]$. In the last decade this picture has become fully proved thanks to the upcoming of a set of precise experiments. In particular, the results obtained with solar and atmospheric neutrinos have been confirmed in experiments using terrestrial beams of neutrinos produced in nuclear reactors and accelerators facilities [3]. The minimum joint description of all the neutrino data requires mixing among all the three known neutrinos $\left(\nu_{e}, \nu_{\mu}, \nu_{\tau}\right)$, which can be expressed as quantum superpositions of three massive states $\nu_{i}$ $(i=1,2,3)$ with masses $m_{i}$. This implies the presence of a leptonic mixing matrix in the weak charged current interactions $[4,5]$ which can be parametrized as:

$$
U_{\mathrm{vac}}=\left(\begin{array}{ccl}
c_{12} c_{13} & s_{12} c_{13} & s_{13} e^{-i \delta_{\mathrm{CP}}} \\
-s_{12} c_{23}-c_{12} s_{13} s_{23} e^{i \delta_{\mathrm{CP}}} & c_{12} c_{23}-s_{12} s_{13} s_{23} e^{i \delta_{\mathrm{CP}}} & c_{13} s_{23} \\
s_{12} s_{23}-c_{12} s_{13} c_{23} e^{i \delta_{\mathrm{CP}}} & -c_{12} s_{23}-s_{12} s_{13} c_{23} e^{i \delta_{\mathrm{CP}}} & c_{13} c_{23}
\end{array}\right),
$$

where $c_{i j} \equiv \cos \theta_{i j}$ and $s_{i j} \equiv \sin \theta_{i j}$. In addition to the Dirac-type phase $\delta_{\mathrm{CP}}$, analogous to that of the quark sector, there are two physical phases associated to the Majorana character of neutrinos, which are not relevant for neutrino oscillations $[6,7]$ and which are therefore omitted in the neutrino oscillation analysis.

In the simplest quantum-mechanical picture, flavor oscillations are generated by the kinematical Hamiltonian for this ensemble $H_{\text {vac }}$ which in the flavor basis $\left(\nu_{e}, \nu_{\mu}, \nu_{\tau}\right)$ reads

$$
H_{\mathrm{vac}}=U_{\mathrm{vac}} D_{\mathrm{vac}} U_{\mathrm{vac}}^{\dagger} \quad \text { with } \quad D_{\mathrm{vac}}=\frac{1}{2 E_{\nu}} \operatorname{diag}\left(0, \Delta m_{21}^{2}, \Delta m_{31}^{2}\right)
$$


The quantities $\Delta m_{21}^{2},\left|\Delta m_{31}^{2}\right|, \theta_{12}$, and $\theta_{23}$ are relatively well determined by the analysis of solar, atmospheric, reactor and long-baseline (LBL) experiments, while only an upper bound is derived for the mixing angle $\theta_{13}$ and barely nothing is known on the CP phase $\delta_{\mathrm{CP}}$ and on the sign of $\Delta m_{31}^{2}$ [8-11]. Given the observed hierarchy between the solar and atmospheric mass-squared splittings there are two possible non-equivalent orderings for the mass eigenvalues, which are conventionally chosen as

$$
\begin{aligned}
& \Delta m_{21}^{2} \ll\left(\Delta m_{32}^{2} \simeq \Delta m_{31}^{2}\right) \text { with }\left(\Delta m_{31}^{2}>0\right) ; \\
& \Delta m_{21}^{2} \ll\left|\Delta m_{31}^{2} \simeq \Delta m_{32}^{2}\right| \text { with }\left(\Delta m_{31}^{2}<0\right) .
\end{aligned}
$$

As it is customary we refer to the first option, eq. (1.3), as the normal scheme, and to the second one, eq. (1.4), as the inverted scheme; in this form they correspond to the two possible choices of the sign of $\Delta m_{31}^{2} \cdot{ }^{1}$

The flavor evolution of this neutrino ensemble is also affected by the difference in the matter potential induced by neutrino-matter interactions when it propagates in a background of sufficiently dense matter through the so-called Mikheev-Smirnov-Wolfenstein (MSW) mechanism [12, 13]. Within the context of the Standard Model (SM) of particle interactions, this effect is fully determined and leads to a matter potential which for neutral matter is proportional to the number density of electrons in the background $N_{e}(r), V=\sqrt{2} G_{F} N_{e}(r)$, and which only affects electron neutrinos. The evolution of the ensemble is then determined by the Hamiltonian $H^{\nu}=H_{\mathrm{vac}}+H_{\mathrm{mat}}^{\mathrm{SM}}$, with $H_{\mathrm{mat}}^{\mathrm{SM}}=$ $\sqrt{2} G_{F} N_{e}(r) \operatorname{diag}(1,0,0)$. The presence and magnitude of this potential in the propagation of solar neutrinos can be tested in solar neutrino experiments (and in combination with KamLAND), and, as pointed out in ref. [14], it agrees well with the SM prediction. Conversely the effects associated with non-standard forms of the matter potential in solar neutrino propagation have been studied and constrained by the analysis of solar and KamLAND data [15-26].

Matter background effects also affect the evolution of atmospheric and LBL neutrinos when traveling in the Earth. However standard matter effects for atmospheric and LBL neutrino oscillations are suppressed when compared to solar neutrinos. This is so because atmospheric and LBL neutrinos are dominantly $\nu_{\mu}$ 's at the source, the Earth matter density is smaller, and the characteristic length of the neutrino path in matter is shorter. Nevertheless the presence of non-standard matter effects are known to be relevant when discussing the precise determination of the oscillation parameters in the present and near future atmospheric and LBL experiments [27-52].

In this article we address our current knowledge of the size and flavor structure of the matter background effects in the evolution of atmospheric and LBL neutrinos based exclusively on the analysis the present data. In order to do so we introduce in section 2 a generalized phenomenological parametrization of the matter potential allowing for rescaling of its strength from the SM prediction, rotation from the ee sector, and rephasing with respect to $H_{\text {vac. }}$. We also discuss the connection with the matter potential induced by

\footnotetext{
${ }^{1}$ In this convention the angles $\theta_{i j}$ can be taken without loss of generality to lie in the first quadrant, $\theta_{i j} \in[0, \pi / 2]$, and the CP phase $\delta_{\mathrm{CP}} \in[0,2 \pi]$.
} 
non-standard neutrino interactions (NSI) in matter which provide a well-known theoretical framework for this phenomenological parametrization. In section 3 we present the results of our determination of this generalized matter potential from the analysis of atmospheric, LBL and CHOOZ experiments. In particular we will show that in the most general case, the strength of the potential cannot be determined by these data. However its flavor composition is very much constrained. We will also show that the present determination of the neutrino masses and mixing is robust even in this generalized scenario. In section 4 we will discuss the particular case in which no potential is present in the $e \mu$ and $e \tau$ sector. In this case, a strong bound on the strength of the potential arises from the analysis of atmospheric and LBL neutrinos. We will update the results of ref. [3, 36] and we will revisit the claims that within this scenario it is possible to alleviate the tension between the oscillation results for neutrinos and antineutrinos in the MINOS experiment. In section 5 we will draw our conclusions. Some technical details on the choice of parametrization of the matter potential are given in two appendices.

\section{Formalism}

In the three-flavor oscillation picture, the neutrino (and antineutrino) evolution equation reads:

$$
i \frac{d}{d x}\left(\begin{array}{c}
\nu_{e} \\
\nu_{\mu} \\
\nu_{\tau}
\end{array}\right)=H^{\nu}\left(\begin{array}{c}
\nu_{e} \\
\nu_{\mu} \\
\nu_{\tau}
\end{array}\right)
$$

where $x$ is the coordinate along the neutrino trajectory and the Hamiltonian for neutrinos and antineutrinos is:

$$
H^{\nu}=H_{\mathrm{vac}}+H_{\mathrm{mat}} \quad \text { and } \quad H^{\bar{\nu}}=\left(H_{\mathrm{vac}}-H_{\mathrm{mat}}\right)^{*},
$$

with $H_{\text {vac }}$ given in eq. (1.2). In the Standard Model $H_{\text {mat }}$ is fully determined both in its strength and flavor structure to be $H_{\text {mat }}^{\mathrm{SM}}=\sqrt{2} G_{F} N_{e}(r) \operatorname{diag}(1,0,0)$. In this work we generalize the form of the matter potential to be

$$
H_{\text {mat }}=Q_{\text {rel }} U_{\text {mat }} D_{\text {mat }} U_{\text {mat }}^{\dagger} Q_{\text {rel }}^{\dagger} \quad \text { with }\left\{\begin{aligned}
Q_{\text {rel }} & =\operatorname{diag}\left(e^{i \alpha_{1}}, e^{i \alpha_{2}}, e^{-i \alpha_{1}-i \alpha_{2}}\right) \\
U_{\text {mat }} & =R_{12}\left(\varphi_{12}\right) R_{13}\left(\varphi_{13}\right) \\
D_{\text {mat }} & =\sqrt{2} G_{F} N_{e}(r) \operatorname{diag}(\varepsilon, 0,0)
\end{aligned}\right.
$$

where we denote by $R_{i j}\left(\varphi_{i j}\right)$ a rotation of angle $\varphi_{i j}$ in the $i j$ plane. In this parametrization $\varepsilon$ represents a rescaling of the matter potential strength, $\varphi_{12}$ allows for projection of the potential into the $\nu_{\mu}$ flavor and $\varphi_{13}$ allows for its projection into the $\nu_{\tau}$ flavor. The two additional phases $\alpha_{1}$ and $\alpha_{2}$ included in $Q_{\text {rel }}$ are not a feature of neutrino-matter interactions, but rather a relative feature of the vacuum and matter term: they would become unphysical if any of the two terms weren't there. As described in appendix B one can always take $0 \leq \theta_{i j} \leq \pi / 2$ and $\Delta m_{31}^{2} \geq 0$ and the neutrino mass hierarchy is accounted by the sign of $\varepsilon$ : positive for normal ordering, negative for inverted ordering. For the case of real potential $\left(\alpha_{1}=\alpha_{2}=0\right)$ one must consider $-\pi / 2 \leq \varphi_{i j} \leq \pi / 2$, whereas 
for complex NSI (free $\alpha_{1}$ and $\alpha_{2}, 0 \leq \alpha_{i} \leq 2 \pi$ ) it is enough to assume $0 \leq \varphi_{i j} \leq \pi / 2$. In this parametrization the standard $3 \nu$ oscillations in matter are recovered in the limit $\varepsilon= \pm 1$ and $\varphi_{12}=\varphi_{13}=0$, where $\varepsilon=+1$ corresponds to the normal ordering and $\varepsilon=-1$ corresponds to the inverted ordering.

As explained in appendix A (see eq. (A.1) and below) eq. (2.3) is not the most general parametrization for the matter potential, since it has built-in the assumption that two of its eigenvalues are equal. In ref. [37] it was shown that strong cancellations in the oscillation of atmospheric neutrinos occur in this case. Consequently despite our phenomenological parametrization in eq. (2.3) represents only a subspace of the most general parameter space for the matter potential, it is precisely in this subspace where the weakest constraints can be placed.

For the analysis of atmospheric, LBL and CHOOZ data, one can make the additional simplifying assumption of setting $\Delta m_{21}^{2}=0$, hence neglecting the solar splitting. In this limit the $\theta_{12}$ angle and the $\delta_{\mathrm{CP}}$ phase become unphysical, even in the presence of the generalized $H_{\text {mat }}$ introduced above. Thus altogether the relevant flavor transition probabilities for atmospheric, LBL and CHOOZ neutrinos depend on eight parameters: $\left(\Delta m_{31}^{2}, \theta_{13}\right.$, $\left.\theta_{23}\right)$ for the vacuum part, $\left(\varepsilon, \varphi_{12}, \varphi_{13}\right)$ for the matter part, and $\left(\alpha_{1}, \alpha_{2}\right)$ as relative phases.

We notice that within this parametrization and neglecting $\Delta m_{21}^{2}$ both the vacuum and the matter part of the Hamiltonian have each two degenerate eigenvalues. In ref. [53] it was noted that when this occurs the evolution equation can be reduced to an effective twoflavor problem in terms of an intermediate basis. Such effective $2 \times 2$ evolution Hamiltonian depends only on 3 parameters: $\Delta m_{31}^{2}, \varepsilon$, and an angle which is a given function $\theta_{13}, \theta_{23}$, $\varphi_{12}$ and $\varphi_{13}$ (see appendix A for details). Therefore we can solve the evolution in this reduced parameter space and later on project back to the flavor basis. Technically this makes it possible to perform the analysis of the atmospheric, LBL and CHOOZ data in the full eight-dimensional parameter space.

A theoretical framework for our proposed parametrization of the matter potential is provided by NSI affecting neutrino interactions in the Earth matter. They can be described by effective four-fermion operators of the form

$$
\mathcal{L}_{\mathrm{NSI}}=-2 \sqrt{2} G_{F} \varepsilon_{\alpha \beta}^{f P}\left(\overline{\nu_{\alpha}} \gamma^{\mu} \nu_{\beta}\right)\left(\bar{f} \gamma_{\mu} P f\right),
$$

where $f$ is a charged fermion, $P=(L, R)$ and $\varepsilon_{\alpha \beta}^{f P}$ are dimensionless parameters encoding the deviation from standard interactions. Ordinary matter is composed by electrons $(e)$, up-quarks $(u)$ and down-quark $(d)$, and in principle non-standard neutrino-matter interactions can involve any of these particles with different strength. In practice, however, the proton/neutron ratio found in matter is reasonably constant all over the Earth, and pretty close to 1 . This implies that neutrino oscillations are only sensitive to the sum of these interactions, weighted with the relative abundance of each particle. Furthermore NSI enter in neutrino propagation only through the vector couplings $\varepsilon_{\alpha \beta}^{f}=\varepsilon_{\alpha \beta}^{f L}+\varepsilon_{\alpha \beta}^{f R}$. We can therefore define the relevant combination of NSI parameters entering into the neutrino propagation in the Earth as:

$$
\varepsilon_{\alpha \beta} \equiv \sum_{f=e, u, d}\left\langle\frac{N_{f}}{N_{e}}\right\rangle \varepsilon_{\alpha \beta}^{f} \approx \varepsilon_{\alpha \beta}^{e}+3 \varepsilon_{\alpha \beta}^{u}+3 \varepsilon_{\alpha \beta}^{d}
$$


where $N_{f}$ is the number density of the fermion $f$. The corresponding matter Hamiltonian reads:

$$
H_{\mathrm{mat}}=\sqrt{2} G_{F} N_{e}(r)\left(\begin{array}{ccc}
1+\varepsilon_{e e} & \varepsilon_{e \mu} & \varepsilon_{e \tau} \\
\varepsilon_{e \mu}^{*} & \varepsilon_{\mu \mu} & \varepsilon_{\mu \tau} \\
\varepsilon_{e \tau}^{*} & \varepsilon_{\mu \tau}^{*} & \varepsilon_{\tau \tau}
\end{array}\right) .
$$

which includes both the standard (accounted by the " +1 " term in the ee entry) and the non-standard interactions (accounted by the $\varepsilon_{\alpha \beta}$ terms).

The Hermitian matrix $H_{\text {mat }}$ depends on 8 physical parameters. Oscillation experiments are sensitive to the matter potential up to an overall multiple of the identity, thus we can measure two differences of the $\varepsilon_{\alpha \alpha}$ parameters (which must be real) as well as $\varepsilon_{e \mu}, \varepsilon_{e \tau}$ and $\varepsilon_{\mu \tau}$ (which, in general, can be complex). These 8 parameters are reduced to the 5 in eq. (2.3) under the condition that two eigenvalues of $H_{\text {mat }}$ are equal, which eliminates one real parameter, one angle and one phase leading to:

$$
\begin{aligned}
\varepsilon_{e e}-\varepsilon_{\mu \mu} & =\varepsilon\left(\cos ^{2} \varphi_{12}-\sin ^{2} \varphi_{12}\right) \cos ^{2} \varphi_{13}-1, \\
\varepsilon_{\tau \tau}-\varepsilon_{\mu \mu} & =\varepsilon\left(\sin ^{2} \varphi_{13}-\sin ^{2} \varphi_{12} \cos ^{2} \varphi_{13}\right), \\
\varepsilon_{e \mu} & =-\varepsilon \cos \varphi_{12} \sin \varphi_{12} \cos ^{2} \varphi_{13} e^{i\left(\alpha_{1}-\alpha_{2}\right)}, \\
\varepsilon_{e \tau} & =-\varepsilon \cos \varphi_{12} \cos \varphi_{13} \sin \varphi_{13} e^{i\left(2 \alpha_{1}+\alpha_{2}\right)}, \\
\varepsilon_{\mu \tau} & =\varepsilon \sin \varphi_{12} \cos \varphi_{13} \sin \varphi_{13} e^{i\left(\alpha_{1}+2 \alpha_{2}\right)} .
\end{aligned}
$$

Notice that the term " -1 " at the end of $\varepsilon_{e e}-\varepsilon_{\mu \mu}$ accounts for the standard matter term. In terms of the NSI parameters $\varepsilon_{\alpha \beta}$ the magnitude of $\varepsilon$ can be always expressed as:

$$
\begin{aligned}
\varepsilon^{2} & =\frac{3}{2} \min _{\lambda}\left[\sum_{\alpha \beta}\left|\delta_{e \alpha} \delta_{e \beta}+\varepsilon_{\alpha \beta}-\lambda \delta_{\alpha \beta}\right|^{2}\right] \\
& =\frac{1}{2}\left[3 \sum_{\alpha \beta}\left|\delta_{e \alpha} \delta_{e \beta}+\varepsilon_{\alpha \beta}\right|^{2}-\left(1+\sum_{\alpha} \varepsilon_{\alpha \alpha}\right)^{2}\right],
\end{aligned}
$$

and its sign can be defined by the relative size of the eigenvalues of $H_{\text {mat }}$ : $\varepsilon$ is positive (negative) if the two equal eigenvalues are smaller (larger) than the third one.

As mentioned above in ref. [37] it was shown that strong cancellations (hence weak bounds on the $\varepsilon$ 's) in the oscillation of atmospheric neutrinos occur if two of the NSI eigenvalues coincide. This is so because in this limit there is a two-dimensional subspace where $\nu_{\mu} \rightarrow \nu_{\tau}$ oscillations occur as in vacuum. Consequently, although eq. (2.3) cover only a part of the most general parameter space for the matter potential induced by NSI, it is the part where the weakest constraints on NSI can be placed. Consequently our conclusions on the allowed values of the NSI parameters are not expected to be weakened once the "equal eigenvalues" approximation is removed.

We conclude this section by noticing that until recently it was usually assumed that the bounds from loop contributions to flavor changing processes induced by the NSI implied $\varepsilon_{e \mu} \lesssim 10^{-4}$ [54]. If the condition $\varepsilon_{e \mu}=0$ is imposed (together with the assumption that two NSI eigenvalues are equal) one is left with two possible scenarios: (i) $\varepsilon_{\mu \tau}=0$, which is a 
subset of the $e \tau$ sector studied in refs. [37, 38] (who made the assumption $\varepsilon_{e \mu}=\varepsilon_{\mu \tau}=0$ but left both eigenvalues free), and (ii) $\varepsilon_{e \tau}=0$, which corresponds to the NSI being dominantly in the $\mu \tau$ sector $[3,30,36]$ and is revisited in section 4 . However, in ref. [55] it was argued that the loop bound on $\varepsilon_{e \mu}$ does not hold in general and consequently it raised the interest of the general analysis here presented.

\section{Results}

Let us now present the results of a combined analysis of atmospheric, LBL and CHOOZ experiments in the context of oscillations with the generalized matter potential in the Earth in eq. (2.3). It is well known that important constraints on $\nu_{e}$ flavor oscillations driven by $\Delta m_{31}^{2}$ arises from the negative results on $\bar{\nu}_{e}$ disappearance at short baselines at the CHOOZ reactor experiment [56]. We include in our analysis the results of $\mathrm{CHOOZ}$ under the assumption that, because of the short baseline, matter effects are irrelevant in this experiment. For atmospheric neutrinos we include the results of our reanalysis of the Super-Kamiokande I+II+III phases as presented in [57] and described in ref. [9]; details on our simulation of the data samples and the statistical analysis can be found in the appendix of ref. [3]. For what concerns LBL accelerator experiments, we combine the results of the energy spectrum of $\nu_{\mu}$ events obtained by MINOS after an exposure to the Fermilab NuMI beam corresponding to a total of $7.25 \times 10^{20}$ protons on target [58] with the data on antineutrino $\bar{\nu}_{\mu}$ disappearance corresponding to $1.71 \times 10^{20}$ protons on target [59]. We also account for the recent MINOS results on $\nu_{\mu} \rightarrow \nu_{e}$ transitions for which we fit the total event rate of observed $\nu_{e}$ events above the expected background based on an exposure of $7.01 \times 10^{20}$ protons on target [60].

We present the results of the global analysis in figures 1, 2, 3 and 4. For simplicity the results are given for fixed values of the phases $\alpha_{1}=\alpha_{2}=0$. We have verified that the phases have little impact on the results of this analysis. In particular, no information on them can be extracted from the data (i.e., their allowed range is the full $0 \leq \alpha_{i} \leq 2 \pi$ interval) and they have only a minor effect on the determination of the other six real parameters.

Figure 1 contains the three two-dimensional projections of the allowed regions in the oscillation parameters $\left|\Delta m_{31}^{2}\right|, \sin ^{2} \theta_{23}$ and $\sin ^{2} \theta_{13}$ after marginalizing over $\varepsilon, \varphi_{12}$, and $\varphi_{13}$. The regions are shown at $90 \%, 95 \%, 99 \%$ and $3 \sigma$ CL (2 dof). Notice that since we have chosen to absorb the relative sign between the $\Delta m_{31}^{2}$ and the matter potential in $\varepsilon$ there is only one oscillation region. For the sake of comparison we also show in the figure the corresponding regions for the case of standard matter potential with normal ordering $\left(\varepsilon=+1, \varphi_{12}=\varphi_{13}=0\right)$ and inverted ordering $\left(\varepsilon=-1, \varphi_{12}=\varphi_{13}=0\right)$. Figure 2 displays the two-dimensional projections of the allowed regions in the matter potential parameters $\varepsilon, \varphi_{12}$ and $\varphi_{13}$ after marginalizing over $\left|\Delta m_{31}^{2}\right|, \theta_{23}$ and $\theta_{13}$. The regions are shown at $90 \%$, $95 \%, 99 \%$ and $3 \sigma$ CL (2 dof). From these figures we can draw the following conclusions on the determination of the matter potential:

- No bound on the magnitude of the matter effects, $\varepsilon$, can be derived from the analysis of atmospheric and LBL (and CHOOZ) experiments only in this general scenario. 

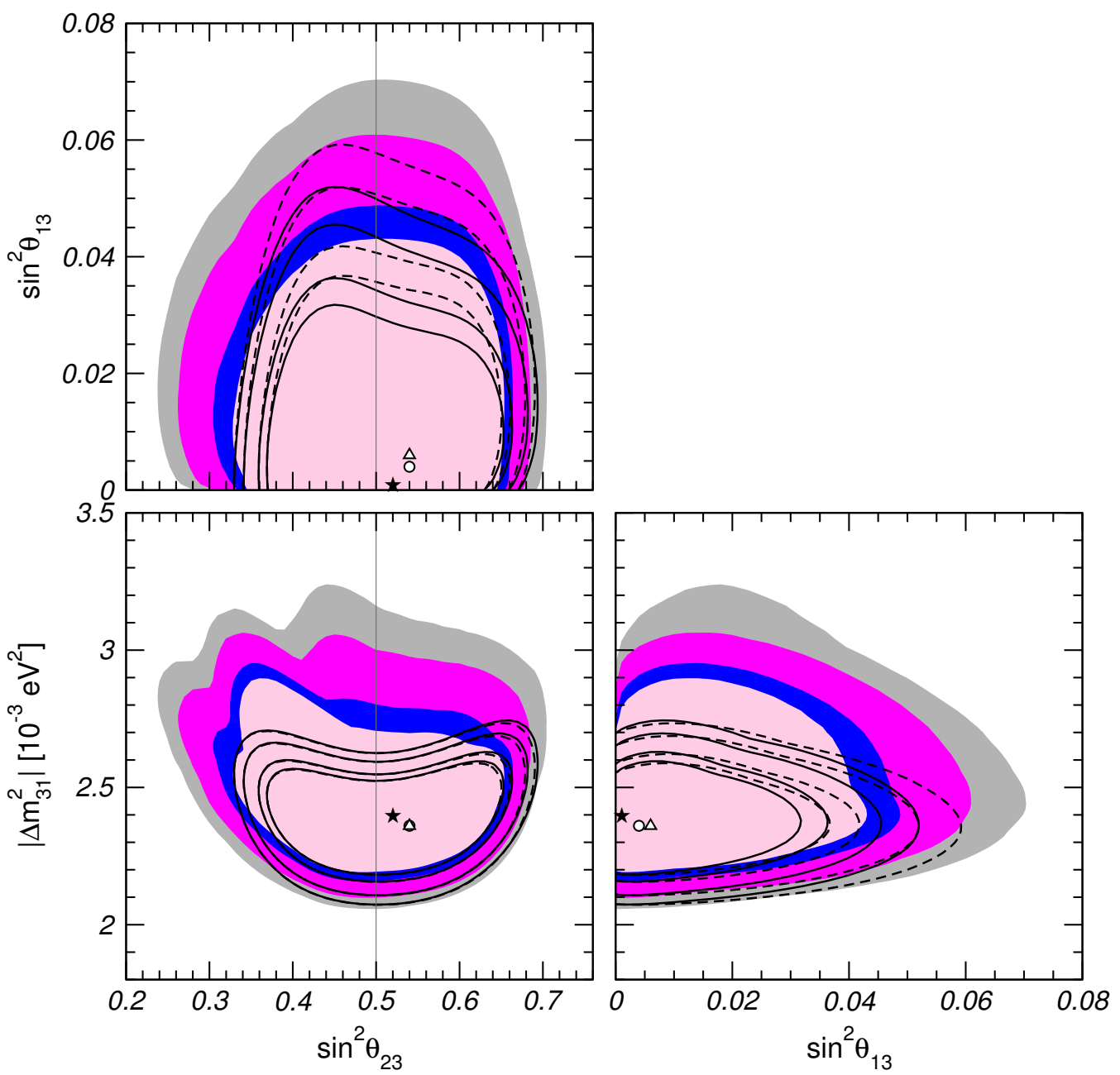

Figure 1. Two-dimensional projections of the allowed regions from the global analysis of atmospheric, LBL and CHOOZ data in the oscillation parameters $\left|\Delta m_{31}^{2}\right|, \sin ^{2} \theta_{23}$ and $\sin ^{2} \theta_{13}$ after marginalizing over $\varepsilon, \varphi_{12}$, and $\varphi_{13}$ (full regions). The best fit point is marked with a star. For the sake of comparison we also show in the figure the corresponding regions for the case of standard matter potential with normal ordering $\left(\varepsilon=+1, \varphi_{12}=\varphi_{13}=0\right)$ as unfilled regions with full lines with best fit point marked with a circle and inverted ordering $\left(\varepsilon=-1, \varphi_{12}=\varphi_{13}=0\right)$ as unfilled regions with dashed lines with best fit point marked with a triangle. The regions are shown at $90 \%$, $95 \%, 99 \%$ and $3 \sigma$ CL (2 dof).

Consequently in order to attain bounds on $\varepsilon$ it is necessary to combine these results with those from other neutrino oscillation samples involving $\nu_{e}$ oscillations, such as solar and KamLAND experiments. However, a bound on $\varepsilon$ can be derived if a certain flavor structure of the matter potential is assumed a priori, implying that $\varphi_{12}$ and/or $\varphi_{13}$ are larger than a certain amount. This is the case, for example, if we assume that no matter effects are present in the $e \mu$ and $e \tau$ projections, which corresponds to $\varphi_{12}=\pi / 2$. We will study this case in the next section.

- Matter potentials with $|\varepsilon| \gtrsim \mathcal{O}(0.2)$ are only allowed as long as their flavor projections out of the ee entry are severely constrained. The constraint is stronger on rotations 


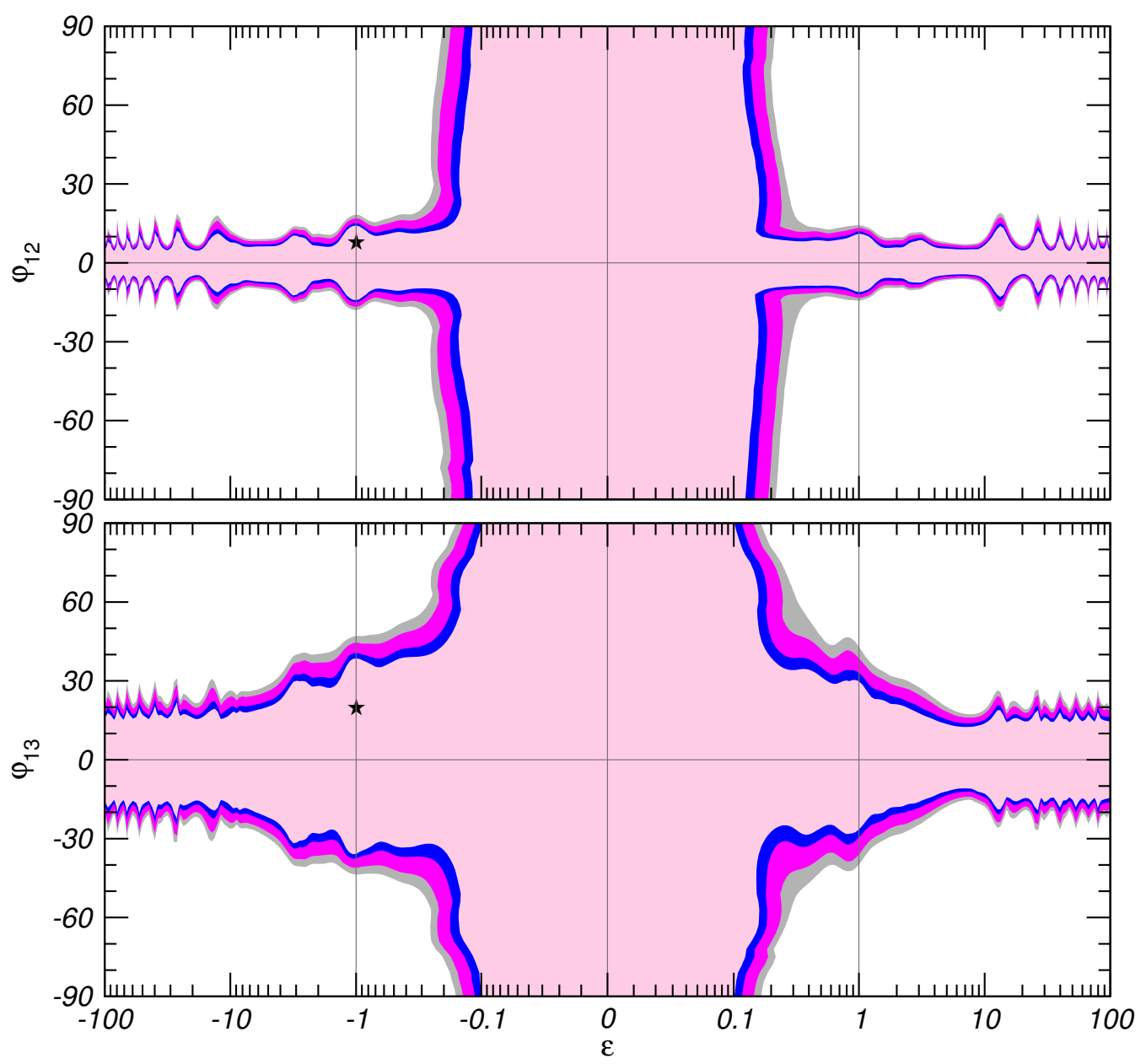

Figure 2. Two-dimensional projection of the of the allowed regions from the global analysis of atmospheric, LBL and $\mathrm{CHOOZ}$ data in the matter potential parameters $\varepsilon, \varphi_{12}$, and $\varphi_{13}$ after marginalization with respect to the undisplayed parameters. The regions are shown at $90 \%, 95 \%$, $99 \%$ and $3 \sigma$ CL (2 dof). The best fit point is marked with a star.

over the $\mu$ flavor, $\varphi_{12}$. We also notice that for large values of $\varepsilon$ the precise bounds on $\varphi_{12}$ and $\varphi_{13}$ present an oscillatory behavior. We will discuss the origin of this beaviour below.

Qualitatively these results can be understood as follows. For $\sqrt{2} G_{F} N_{e}(r) \varepsilon \gg \Delta m_{31}^{2} /\left(2 E_{\nu}\right)$ the neutrinos can undergo flavor oscillations with two different phases:

$$
\begin{aligned}
\tilde{\Delta}_{\text {vac }} & =\frac{\Delta m_{31}^{2} L}{4 E_{\nu}} \times f\left(\theta_{23}, \theta_{13}, \varphi_{12}, \varphi_{13}\right) \\
\tilde{\Delta}_{\text {mat }} & =\frac{\sqrt{2} G_{F} N_{e}(r) \varepsilon L}{2}
\end{aligned}
$$

where $f\left(\theta_{23}, \theta_{13}, \varphi_{12}, \varphi_{13}\right)$ is a combination of trigonometric functions of the four angles: for example, for $\theta_{13}=0$ we get $f=\cos ^{2} \varphi_{12} \cos ^{2} \varphi_{13}+\left(\cos \varphi_{13} \sin \varphi_{12} \cos \theta_{23}-\sin \varphi_{13} \sin \theta_{23}\right)^{2}$. $\nu_{\mu} \leftrightarrow \nu_{\tau}$ transitions oscillate dominantly with phase $\tilde{\Delta}_{\text {vac }}$ : this is the generalization of the 
results in ref. $[37,38]$ and it means that in this scenario $\nu_{\mu} \rightarrow \nu_{\tau}$ oscillations have the same dependence on the neutrino energy and distance as vacuum oscillations, even for large $\varepsilon$, which opens the possibility of giving a good description of atmospheric and LBL results. In the same regime $\nu_{\mu} \leftrightarrow \nu_{e}$ and $\nu_{e} \leftrightarrow \nu_{\tau}$ transitions proceed dominantly via oscillations of phase $\tilde{\Delta}_{\text {mat }}$

$$
\begin{aligned}
& P_{e \mu}=\cos ^{2} \varphi_{13} \sin ^{2}\left(2 \varphi_{12}\right) \sin ^{2} \tilde{\Delta}_{\text {mat }} \\
& P_{e \tau}=\cos ^{2} \varphi_{12} \sin ^{2}\left(2 \varphi_{13}\right) \sin ^{2} \tilde{\Delta}_{\text {mat }}
\end{aligned}
$$

This means that even for $\theta_{13}=0$ atmospheric $\nu_{e}$ 's can disappear into either $\nu_{\mu}$ or $\nu_{\tau}$ and they do so independently of their energy. Similarly, some of the atmospheric muon neutrinos will oscillate into electron neutrinos independently of their energy. This is in clear conflict with the atmospheric data and therefore the amplitudes of these oscillations are constrained. This leads to the strong bounds on $\left|\varphi_{12}\right|$ and $\left|\varphi_{13}\right|$ observed in figure 2 for $|\varepsilon| \gtrsim \mathcal{O}(0.2)$. Furthermore, since the atmospheric $\nu_{e}$ data mostly constrain the sum of $P_{e \mu}+P_{e \tau}$, the bounds on $\varphi_{12}$ and $\varphi_{13}$ for a given $\varepsilon$ are strongly correlated. Notice also that atmospheric neutrino angular bins correspond to given ranges of $L$, rather than just a specific value as in the case of MINOS, so for large $\varepsilon$ the phase $\tilde{\Delta}_{m}$ is averaged in $L$ and the atmospheric bounds on $\varphi_{12}$ and $\varphi_{13}$ become independent of $\varepsilon$.

Similarly to the atmospheric neutrino data, the MINOS $\nu_{\mu}$ disappearance spectrum is well explained by the vacuum-like oscillations $\nu_{\mu} \rightarrow \nu_{\tau}$. But it also leads to further constraints on the contribution from $P_{e \mu}$ in (3.3), since the presence of this term would induce an energy independent contribution to the $\nu_{\mu}$ survival probability which is in conflict with the observations. This tightens the atmospheric constraint on $\varphi_{12}$. Since $L$ in MINOS is fixed, the contribution of $P_{e \mu}$ to the MINOS spectrum has an oscillatory behavior on $\varepsilon$ (i.e., the phase $\tilde{\Delta}_{m}$ is not averaged over $L$ as long as the size of the detector is small compared to $\left[G_{F} N_{e}(r) \varepsilon\right]^{-1}$ ) which leads to the "spike-like" shape of the allowed region for $\varphi_{12}$ versus $\varepsilon$. Given the strong correlation between the allowed values of $\varphi_{13}$ and $\varphi_{12}$ imposed by the atmospheric data, this "spike-like" shape is also projected in the allowed region for $\varphi_{13}$ versus $\varepsilon$.

For what concerns the standard oscillation parameters, our analysis shows that their present determination is rather robust even in the presence of a generalized matter potential of the form (2.3). To further illustrate this we show in figure 3 the allowed regions of the three oscillation parameters as a function of the matter potential magnitude $\varepsilon$. As seen in the figure, the allowed ranges for the oscillation parameters do not get substantially modified for any value of $\varepsilon$ (see also eq. (5.1)).

The dependence on these results on the data samples included in the analysis is shown in figure 4 where we plot the one dimensional projections of the $\Delta \chi^{2}$ for three different combination of experiments as a function of each of the six parameters. In each case the $\Delta \chi^{2}$ is defined with respect to the minimum obtained for the standard oscillation case which occurs in the normal ordering $\left(\varepsilon=+1, \varphi_{12}=\varphi_{13}=0\right)$. We read from the figure that for any of the three combination of data shown, there is no substantial improvement of the fit due to the inclusion of the extra parameters. $\Delta \chi^{2}$ is reduced by a maximum of 1.5 units 


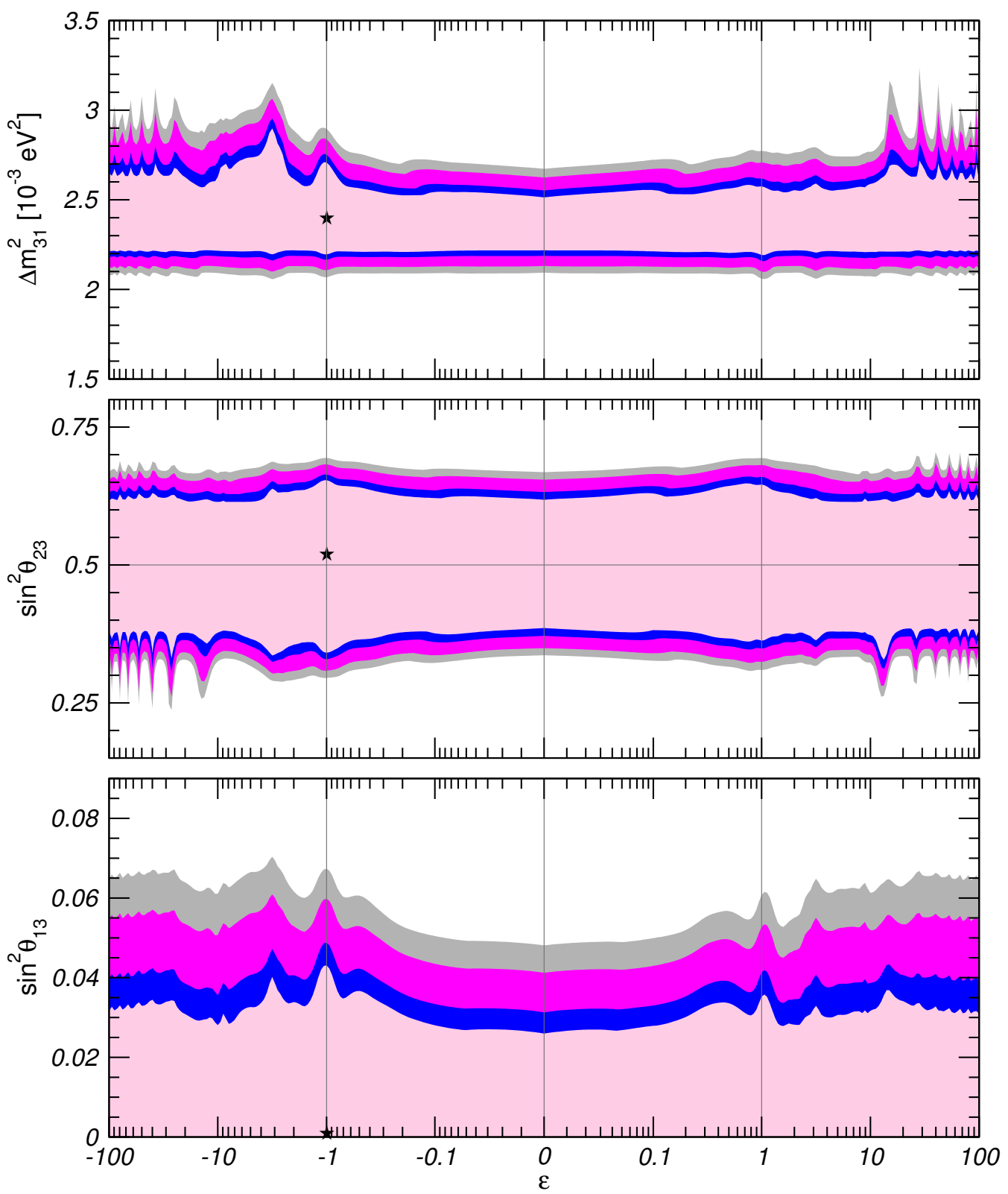

Figure 3. Two-dimensional projection of the of the allowed regions from the global analysis of atmospheric, LBL and CHOOZ for the oscillation parameters and the $\varepsilon$ parameter of the matter potential magnitude. The regions are shown at 90\%, 95\%,99\% and $3 \sigma$ CL ( 2 dof). The best fit point is marked with a star.

in the global analysis. In other words, even though no constraint on the magnitude of the matter effects can be placed by the data, the data do not show any need of a non-standard matter potential. In particular comparing the results including and not including the $\bar{\nu}_{\mu}$ MINOS results we see that inclusion of non-standard matter effects does not result into a substantial improvement on the quality of the combined fit. From the lower panel of the figure we see that inclusion of the MINOS antineutrino results implies the appearance of a 

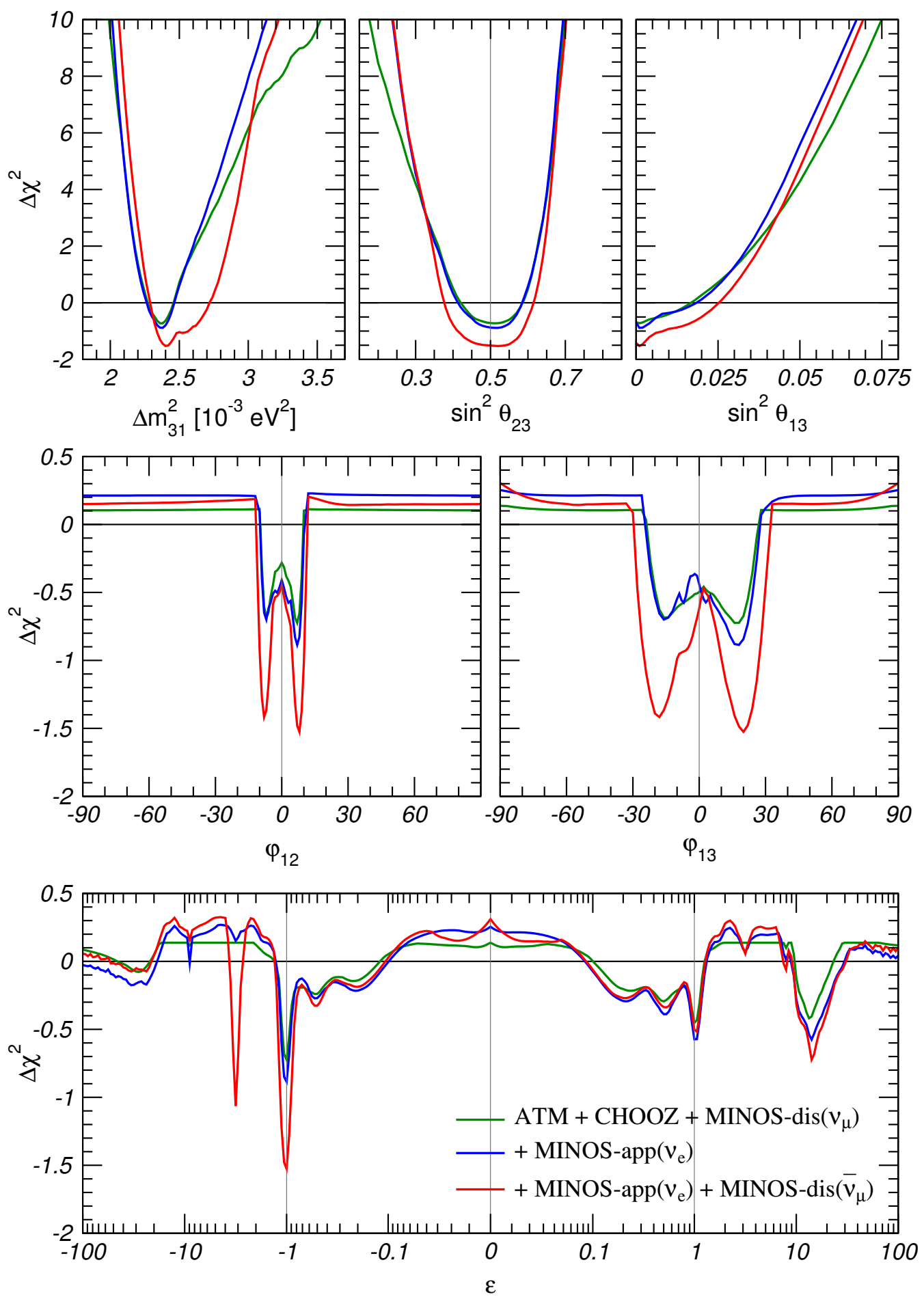

Figure 4. Dependence of the $\Delta \chi^{2}$ function for different combination of observables on the oscillation and matter potential parameters. In each panel the $\Delta \chi^{2}$ function has been marginalized with respect to all other parameters. The $\Delta \chi^{2}$ is defined with respect to the minimum for pure oscillations which lies in the $\varepsilon=+1, \varphi_{12}=\varphi_{13}=0$ subspace. 
new local minimum at $\varepsilon=-3$. This local minimum occurs at $\varphi_{13}=-16^{\circ}$ and $\varphi_{12}=-7^{\circ}$ and it is the equivalent of the best solution found in the partial analysis of refs. [61-63] to explain the discrepancy of MINOS neutrino and antineutrino results in the framework of NSI. However, as seen in the figure, we find that the "improvement" on the quality of the description associated with this new minimum is about 1 unit in the global $\chi^{2}$. This is partly due to the constraints from atmospheric neutrino data on the required values of the NSI and partly on details associated with the statistical analysis of MINOS in the presence of NSI, which we will quantify in more detail in the next section.

As a matter of curiosity we notice that the best fit of the global analysis corresponds to "standard" magnitude matter effects with inverted mass ordering $(\varepsilon=-1)$, maximal $\theta_{23}$ and $\theta_{13}=0$, but with non-standard flavor projections $\varphi_{12}=8^{\circ}$ and $\varphi_{13}=20^{\circ}$. This behavior is already present when only atmospheric and $\mathrm{CHOOZ}$ data are included in the analysis and it is driven by the small excess of $\nu_{e}$ events in the multi-GeV atmospheric samples. This excess was more significant in SK-I and was highlighted in ref. [64] as a possible "hint" for non-zero $\theta_{13}$, although as pointed out in refs. $[9,11]$ the statistical significance of this effect was reduced in SK-II and SK-III samples. What we find in our analysis is that it is slightly easier to fit the data with a non-standard flavor structure of the matter potential and a zero $\theta_{13}$ than with standard matter potential and a non-vanishing $\theta_{13}$. Nevertheless the difference, yielding a $\Delta \chi^{2}=-1.5$, is not statistically significant.

Finally we show in figure 5 the two-dimensional allowed regions projected in terms of the equivalent NSI parameters $\varepsilon_{\alpha \beta}$, see eq. (2.7). The figure illustrates that although no absolute bound can be place on the individual $\varepsilon_{\alpha \beta}$ parameters, they can only be large if they are highly correlated among them. In other words, in order to comply with the observed data in atmospheric, LBL and CHOOZ experiments, a beyond the SM scenario which could generate such large NSI parameters must have a very constrained flavor structure which implies, for example, $\varepsilon_{\mu \tau} / \varepsilon \lesssim 0.1$ which, unfortunately, is not the one required to explain the MINOS $\nu_{\mu}$ versus $\bar{\nu}_{\mu}$ results. We elaborate more on this in the following section.

\section{$4 \quad$ No matter effects in $e \mu$ and $e \tau$ sectors}

In this section we study the particular case in which no potential is present in the $e \mu$ and $e \tau$ sector, or, equivalently, that the NSI parameters $\varepsilon_{e \mu}=\varepsilon_{e \tau}=0$ are known to be severely constrained from other sectors. As described in refs. [30, 36], in this case a bound on the strength of the potential arises from the analysis of atmospheric and LBL neutrinos. From eq. (2.7) we see that $\varepsilon_{e \mu}=\varepsilon_{e \tau}=0$ corresponds to $\varphi_{12}=\pi / 2$. If we further assume $\theta_{13}=0$ then the $\nu_{e}$ decouples from the evolution equation. Moreover one of the two phases become unphysical so that the matter part of the Hamiltonian depends on three parameters. In refs. $[3,36]$ we labeled them $\mathcal{F}, \xi$ and $\eta$ and they are related to the present notation by

$$
\mathcal{F}=\sqrt{\left|\varepsilon_{\mu \tau}\right|^{2}+\frac{\left(\varepsilon_{\mu \mu}-\varepsilon_{\tau \tau}\right)^{2}}{4}}=\frac{\varepsilon}{2}, \quad \xi=\frac{\left|\varepsilon_{\mu \tau}\right|}{\mathcal{F}}=\varphi_{13}, \quad \eta=\arg \left(\varepsilon_{\mu \tau}\right)=\alpha_{1}+2 \alpha_{2} .
$$

The relevant oscillation probabilities are given by

$$
P_{\nu_{\mu} \rightarrow \nu_{\mu}}=1-P_{\nu_{\mu} \rightarrow \nu_{\tau}}=1-\sin ^{2} 2 \Theta \sin ^{2}\left(\frac{\Delta m_{31}^{2} L}{4 E_{\nu}} \mathcal{R}\right) .
$$



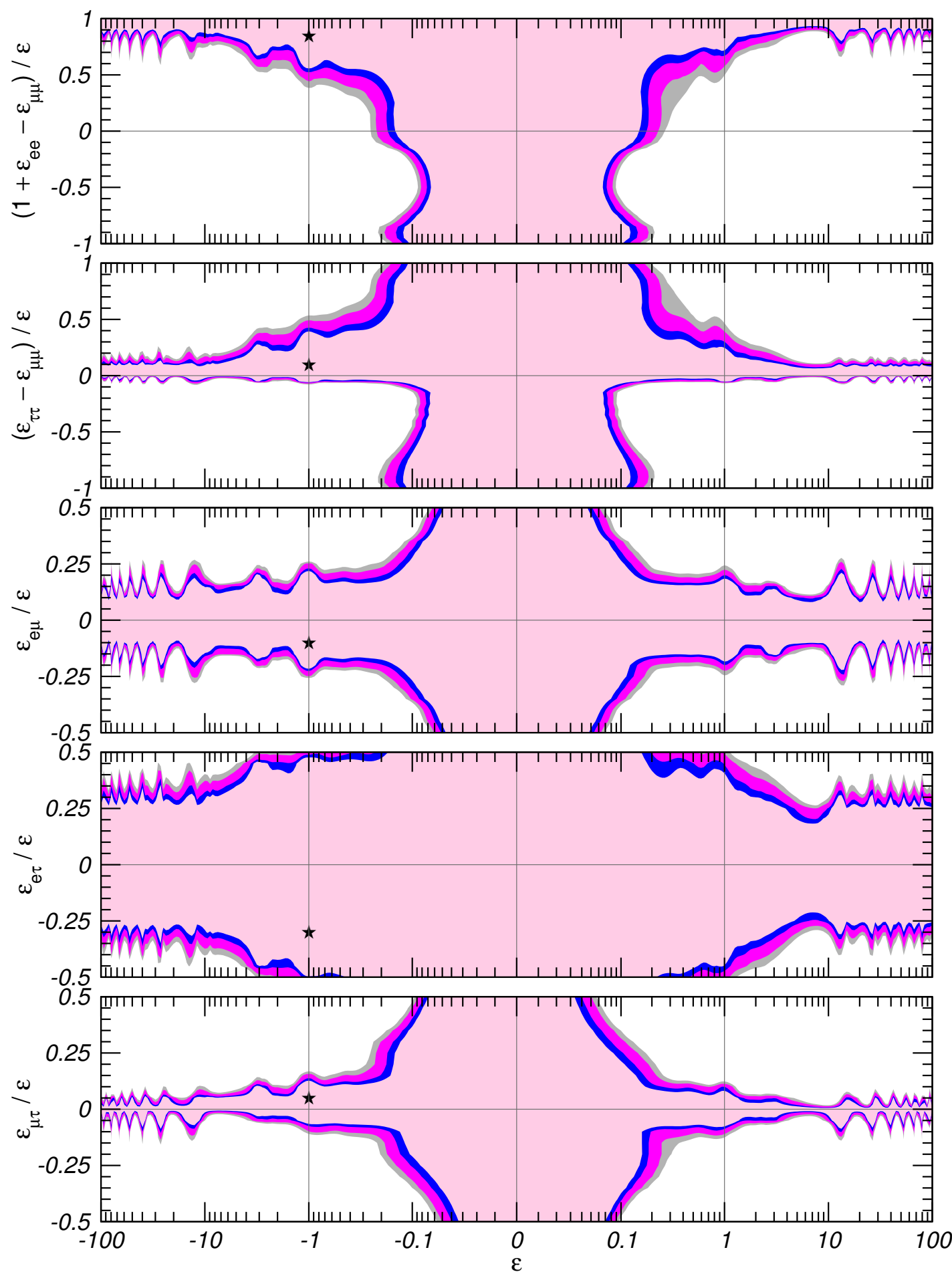

Figure 5. Two-dimensional projection of the of the allowed regions from the global analysis of atmospheric, LBL and $\mathrm{CHOOZ}$ in terms of the NSI parameters $\varepsilon_{\alpha \beta}$ versus the $\varepsilon$ parameter. The regions are shown at $90 \%, 95 \%, 99 \%$ and $3 \sigma$ CL (2 dof). The best fit point is marked with a star. 
where

$$
\begin{aligned}
\sin ^{2} 2 \Theta & =\frac{1}{\mathcal{R}^{2}}\left(\sin ^{2} 2 \theta_{23}+R^{2} \sin ^{2} 2 \varphi_{13}+2 R \sin 2 \theta_{23} \sin 2 \varphi_{13} \cos \eta\right) \\
\mathcal{R} & =\sqrt{1+R^{2}+2 R\left(\cos 2 \theta_{23} \cos 2 \varphi_{13}+\sin 2 \theta_{23} \sin 2 \varphi_{13} \cos \eta\right)}, \\
R & =\frac{\sqrt{2} G_{F} N_{e}(r) \varepsilon L}{2} \cdot \frac{4 E_{\nu}}{\Delta m_{31}^{2}} .
\end{aligned}
$$

So in this case, for large $\varepsilon$ the unique oscillation wavelength becomes energy independent in contradiction with the data, and an upper bound on the magnitude of the non-standard matter effects can be placed. Also, since in this case we are fixing $\varphi_{12}=\pi / 2$ the standard two-neutrino $\nu_{\mu} \rightarrow \nu_{\tau}$ oscillation scenario is recovered in the limit $\varepsilon=0$.

In figure 6 we show the two-dimensional projections of the allowed parameter region for the analysis of atmospheric and LBL data. The filled areas in the upper left panel show the allowed regions on the $\left(\Delta m_{31}^{2}, \sin ^{2} \theta_{23}\right)$ plane. For the sake of comparison we also show the lines corresponding to the contours in the absence of new physics. We see that in this constrained scenario the determination of the oscillation parameters is very much unaffected by the presence of the non-standard matter effects. The other panels of figure 6 show different projections of the allowed values of the parameters characterizing the strength and mixing of the non-standard matter effects. The solid contours correspond to arbitrary values of the phase $\eta$, while the colored regions correspond to the case $\eta \in\{0, \pi\}$. Translated in terms of NSI the corresponding $90 \%(3 \sigma)$ bounds read:

$$
(-0.055)-0.035 \leq \varepsilon_{\mu \tau} \leq+0.018(+0.035), \quad\left|\varepsilon_{\tau \tau}-\varepsilon_{\mu \mu}\right| \leq 0.097(0.16)
$$

for real NSI, and

$$
\left|\varepsilon_{\mu \tau}\right| \leq 0.035(0.055), \quad\left|\varepsilon_{\tau \tau}-\varepsilon_{\mu \mu}\right| \leq 0.11(0.18)
$$

for the general case of complex $\varepsilon_{\mu \tau}$. For the sake of comparison we show in the two lower panels of figure 6 the corresponding regions obtained in the general analysis in figure 5 as dashed contours. We see how only in the restricted analysis a bound on the $\varepsilon$ parameter is set. However the corresponding flavor projections are not more severely constrained in this restricted analysis when compared to the general one presented in the previous section.

To finish this section we turn to the possible role of non-vanishing $\varepsilon_{\mu \tau}$ and $\varepsilon_{\tau \tau}-\varepsilon_{\mu \mu}$ to resolve the tension between the oscillation results for neutrinos and antineutrinos in the MINOS experiment [58, 59]. As shown in the previous section, even in the most general case we found that the inclusion of the matter potential leads to a decrease of the $\Delta \chi^{2}$ of at most $\sim 1.5$ units with respect to pure oscillations. To further quantify the effect of NSI in the $\mu \tau$ sector in the MINOS data we show in figure 7 (right panel) the $\Delta \chi^{2}$ of the analysis of MINOS neutrino and antineutrino results in the framework of $\nu_{\mu} \rightarrow \nu_{\tau}$ oscillations in the presence of non-vanishing NSI in the $\mu \tau$ sector. For the sake of comparison with the literature we show the results for two analysis, one in which all the data of all energy bins of the MINOS neutrino and antineutrino spectrum are included and one where only the bins with $1<E_{\nu}<5 \mathrm{GeV}$ and $1<E_{\bar{\nu}}<8 \mathrm{GeV}$ are included 

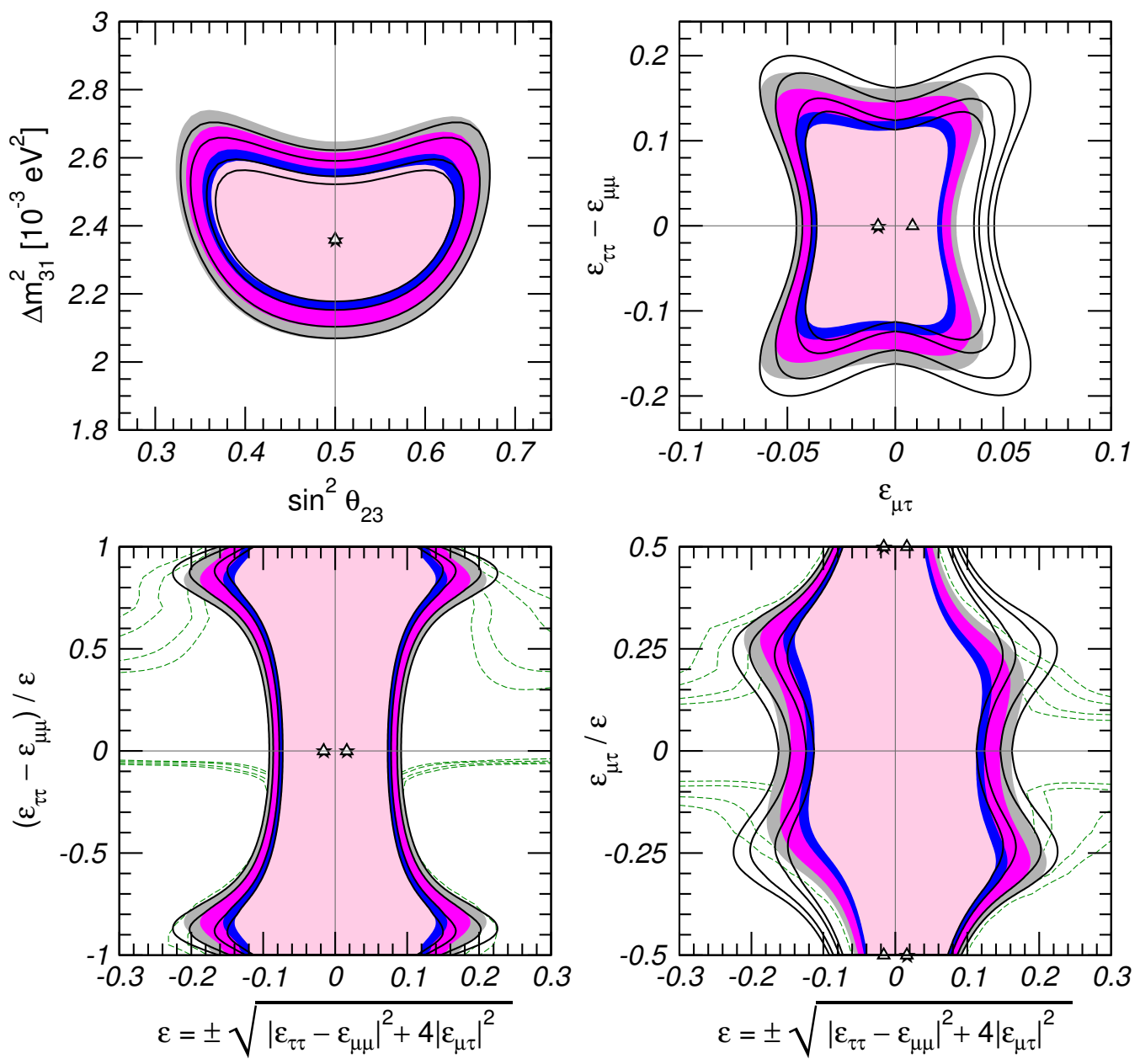

Figure 6. Allowed parameter regions for the analysis of atmospheric and LBL data in presence of $\nu_{\mu} \rightarrow \nu_{\tau}$ oscillations and non-standard matter effects. Each panel shows a two-dimensional projection of the allowed five-dimensional region after marginalization with respect to the three undisplayed parameters. The different contours correspond to the two-dimensional allowed regions at $90 \%, 95 \%, 99 \%$ and $3 \sigma$ CL (2 dof). The filled areas in the upper left panel show the projected two-dimensional allowed regions on the $\left(\Delta m_{31}^{2}, \sin ^{2} \theta_{23}\right)$ plane. The best fit point is marked with a star. For the sake of comparison we also show the lines corresponding to the contours in the absence of new physics and mark with a triangle the position of the best fit point. The regions in other panels show different projections of the allowed values of the parameters characterizing the strength and mixing of the new matter effects. The full regions correspond to the case $\eta \in\{0, \pi\}$ with best fit point marked by a star, while the solid lines correspond to arbitrary values of the phase $\eta$ with best fit point marked by a triangle. In the two lower panels we show for comparison the corresponding regions obtained in the general analysis in figure 5 (dashed contours).

as in ref. [61]. We also show in the left panel of figure 7 the corresponding oscillation regions for the analysis of MINOS in the framework of pure $\Delta m_{31}^{2} \nu_{\mu} \rightarrow \nu_{\tau}$ oscillations for the two analysis. As seen in the figure, in the framework of $\Delta m_{31}^{2}$ oscillations only both analysis yield equivalent oscillation regions. This is so because for pure $\Delta m_{31}^{2}$ oscillations the data of the higher energy bins has little relevance in the determination of the oscillation 

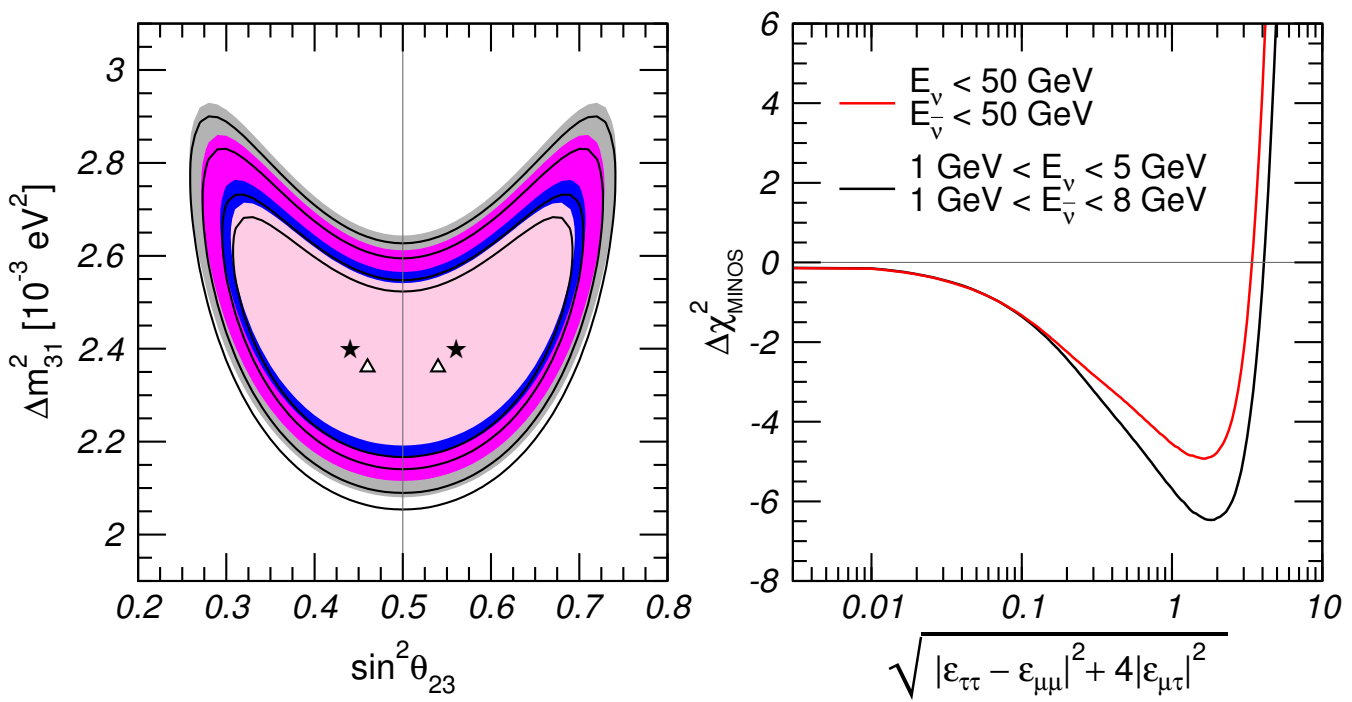

Figure 7. Results of the analysis of MINOS $\nu_{\mu}$ and $\bar{\nu}_{\mu}$ disappearance data for two sets of analysis differing in the inclusion of the high energy bins (see text for details). The left panel show the allowed regions in the framework of pure $\Delta m_{31}^{2}$-driven oscillations. The full regions are the twodimensional allowed regions at 90\%, 95\%, 99\% and $3 \sigma$ CL (2 dof) for the analysis including all the bins and the corresponding best fit point is marked with a star. The unfilled regions correspond to the analysis where only the $1<E_{\nu}<5 \mathrm{GeV}$ and $1<E_{\bar{\nu}}<8 \mathrm{GeV}$ bins are considered (best fit point marked with a triangle). The right panel show the $\Delta \chi_{\text {MINOS }}^{2}$ as a function of the NSI strength for the five-parameter analysis (in terms of oscillations plus non-standard matter effects in the $\mu \tau$ sector) after marginalizing with respect to to the four undisplayed parameters. The lower (upper) curve corresponds to the analysis without (with) inclusion of the high energy bins.

parameters since at those energies the $\Delta m_{31}^{2}$-driven oscillation wavelength is much longer than the characteristic $L$ in MINOS so the corresponding survival probability is 1 . However, when NSI are included, the $\nu_{\mu}$ and $\bar{\nu}_{\mu}$ survival probabilities acquire an energy independent piece as seen in eq. (4.2). As a consequence, in the presence of NSI the inclusion of the high energy bins is relevant to the conclusions of the analysis. This is quantified in the right panel of figure 7 where we show the $\Delta \chi_{\text {MINOS }}^{2}$ for the five-parameter analysis (in terms of oscillations plus non-standard matter effects in the $\mu \tau$ sector) as a function of the strength parameter for the non-standard matter potential after marginalizing with respect to the other four parameters $\left(\Delta m_{31}^{2}, \theta_{23}, \varphi_{13}, \eta\right)$. Here $\Delta \chi_{\text {MINOS }}^{2}$ is defined with respect to the pure oscillation scenario. From this figure we read than when only the low energy bins are included, NSI seem to be able to improve the agreement between $\nu_{\mu}$ and $\bar{\nu}_{\mu}$ disappearance data by $\Delta \chi_{\text {MINOS }}^{2} \simeq-7$, in good agreement with the results in ref. [61]. However the inclusion of the effect of these same NSI in the high energy bins spoils this improvement and leads only to $\Delta \chi_{\text {MINOS }}^{2} \simeq-5$. Furthermore the required strength for the NSI parameters is in conflict with the atmospheric results, as shown in the previous section and correctly noted in [61]. 


\section{Summary}

In this work we have addressed the question: what do atmospheric and LBL neutrino data teach us about the presence and flavor structure of matter effects in propagation?

First, to set out to answer this question we have introduced in section 2 a phenomenological parametrization of the matter potential allowing for departures of the SM one by rescaling of its strength, rotation from the ee sector, and rephasing with respect to vacuum. In this scenario after setting $\Delta m_{21}^{2}=0$ in the analysis of atmospheric, LBL and CHOOZ data, the relevant flavor transition probabilities depend on eight parameters: the three oscillation parameters $\left(\Delta m_{31}^{2}, \theta_{23}\right.$ and $\left.\theta_{13}\right)$, three matter potential parameters ( $\varepsilon$ which represents a rescaling of the matter potential strength, $\varphi_{12}$ which allows for projection of the potential into the $\nu_{\mu}$ flavor, and $\varphi_{13}$ which allows for its projection into the $\nu_{\tau}$ flavor) and two relative phases. The relation to the matter potential induced by NSI is given in eq. (2.7).

The answer to the question in the most general case is given in section 3 . We find that in general the strength of the matter potential $\varepsilon$ cannot be determined by the analysis. This is the generalization of the results in ref. [37, 38] and it is due to the fact that for $\sqrt{2} G_{F} N_{e}(r) \varepsilon \gg \Delta m_{31}^{2} /\left(2 E_{\nu}\right), \nu_{\mu} \leftrightarrow \nu_{\tau}$ transitions oscillate dominantly with the phase $\tilde{\Delta}_{\text {vac }}$ in eq. (3.1) and have the same dependence on the neutrino energy and distance as vacuum oscillations. This allows for good description of atmospheric and LBL results even for very large values of $\varepsilon$.

However important information is obtained from the data analysis on the flavor structure of the matter potential. This is so because for large $\varepsilon$ the $\nu_{\mu} \leftrightarrow \nu_{e}$ (eq. (3.3)) and $\nu_{e} \leftrightarrow \nu_{\tau}$ (eq. (3.4)) transitions proceed dominantly via oscillations with the energy independent phase $\tilde{\Delta}_{\text {mat }}$ in eqs. (3.2) and amplitudes controlled by the projection angles $\varphi_{12}$ and $\varphi_{13}$. This means that even for $\theta_{13}=0$ atmospheric $\nu_{e}$ 's can disappear into either $\nu_{\mu}$ or $\nu_{\tau}$ and they do so independently of their energy. Similarly, some atmospheric and LBL $\nu_{\mu}$ 's will oscillate into electron neutrinos independently of their energy. This is in clear conflict with the atmospheric and LBL data, and as a consequence potentials with $|\varepsilon| \gtrsim \mathcal{O}(0.2)$ are only allowed as long as their flavor projections out of the ee entry are severely constrained. As can be seen in figure 2, the constraints are stronger on rotations over the $\mu$ flavor, controlled by $\varphi_{12}$.

We also find that the present determination of the oscillation parameters is rather robust even in the presence of this general form of the matter potential as illustrated in figures 1 and 3 . For example the $3 \sigma$ ranges of the oscillation parameters read:

$$
\begin{array}{rrr}
\text { Oscillations } & \text { Oscillations }+ \text { Generalized Matter Potential } \\
2.11 \times 10^{-3} \leq\left|\Delta m_{31}^{2}\right| \leq 2.69 \times 10^{-3} & 2.10 \times 10^{-3} \leq\left|\Delta m_{31}^{2}\right| \leq 3.06 \times 10^{-3} \\
0.34 \leq \sin ^{2} \theta_{23} \leq 0.68 & 0.26 \leq \sin ^{2} \theta_{23} \leq 0.68 \\
\sin ^{2} \theta_{13} \leq 0.051 & \sin ^{2} \theta_{13} \leq 0.060
\end{array}
$$

Concerning the quality of the fit, we find that even though no constraint on the magnitude of the matter effects can be placed by the data, the data does not show any favoring for a non-standard matter potential. There is no substantial improvement on the statistical 
quality of the fit due to the inclusion of the extra matter parameters with $\Delta \chi^{2}$ reduced by a maximum of 1.5 units in the global analysis. This is so even after the inclusion of the MINOS $\bar{\nu}_{\mu}$ disappearance results. Thus we conclude that within the context of a global analysis the inclusion of the NSI does not significantly alleviate the tension between the oscillation results for neutrinos and antineutrinos arising from the MINOS $\bar{\nu}_{\mu}$ disappearance results. This is partly due to the constraints from atmospheric neutrino data and partly to the fact that the non-standard matter potential parameters required to better fit the MINOS energy spectrum of $\nu_{\mu}$ and $\bar{\nu}_{\mu}$ events with $E_{\nu} \leq 5 \mathrm{GeV}$ and $E_{\bar{\nu}} \leq 8 \mathrm{GeV}$ with the same oscillation parameters worsens the description of the spectrum of $E_{\nu}>5 \mathrm{GeV}$ events as shown in the right panel of figure 7 .

Finally we have revisited the particular scenario in which matter effects in the $e \mu$ and $e \tau$ sectors are very suppressed (section 4). In this case a bound on the strength of the NSI in the $\mu \tau$ sector can be set. The corresponding updated bounds shown in eqs. (4.6) and (4.7) are 5-20\% stronger than our last published bounds in ref. [3].

\section{Acknowledgments}

We thank O. Peres for comments. This work is supported by Spanish MICINN grants 2007-66665-C02-01, FPA-2009-08958, FPA-2009-09017 and consolider-ingenio 2010 grant CSD-2008-0037, by CUR Generalitat de Catalunya grant 2009SGR502, by Comunidad Autonoma de Madrid through the HEPHACOS project S2009/ESP-1473, by USA-NSF grant PHY-0969739 and by EU grant EURONU.

\section{A Parametrization of the matter potential}

We give here some details on the simplifications associated with the condition that both the vacuum and the matter part of the Hamiltonian have each two degenerate eigenvalues. In the most general case the matter potential can be parametrized as:

$$
H_{\text {mat }}=Q_{\text {rel }} U_{\text {mat }} D_{\text {mat }} U_{\text {mat }}^{\dagger} Q_{\text {rel }}^{\dagger} \text { with }\left\{\begin{aligned}
Q_{\text {rel }} & =\operatorname{diag}\left(e^{i \alpha_{1}}, e^{i \alpha_{2}}, e^{-i \alpha_{1}-i \alpha_{2}}\right), \\
U_{\text {mat }} & =R_{12}\left(\varphi_{12}\right) \tilde{R}_{13}\left(\varphi_{13}, \delta_{\mathrm{NS}}\right) R_{23}\left(\varphi_{23}\right), \\
D_{\text {mat }} & =\sqrt{2} G_{F} N_{e}(r) \operatorname{diag}\left(\varepsilon, \varepsilon^{\prime}, 0\right)
\end{aligned}\right.
$$

Setting $\varepsilon^{\prime}=0$ implies that the $\varphi_{23}$ angle and the $\delta_{\text {NS }}$ phase become unphysical. In the limit $\Delta m_{21}^{2}=0$ the general Hamiltonian reduces to:

$$
H=R_{23}^{\theta} R_{13}^{\theta} D_{\text {vac }} R_{13}^{\theta \dagger} R_{23}^{\theta \dagger}+Q_{\text {rel }} R_{12}^{\varphi} R_{13}^{\varphi} D_{\text {mat }} R_{13}^{\varphi \dagger} R_{12}^{\varphi \dagger} Q_{\text {rel }}^{\dagger}
$$

where $R_{i j}^{\theta} \equiv R_{i j}\left(\theta_{i j}\right)$ and $R_{i j}^{\varphi} \equiv R_{i j}\left(\varphi_{i j}\right)$. We can write:

$$
H=R_{23}^{\theta} R_{13}^{\theta}\left[D_{\mathrm{vac}}+\left(R_{13}^{\theta \dagger} R_{23}^{\theta \dagger} Q_{\mathrm{rel}} R_{12}^{\varphi} R_{13}^{\varphi}\right) D_{\text {mat }}\left(R_{13}^{\varphi \dagger} R_{12}^{\varphi \dagger} Q_{\text {rel }}^{\dagger} R_{23}^{\theta} R_{13}^{\theta}\right)\right] R_{13}^{\theta \dagger} R_{23}^{\theta \dagger}
$$

where we simply collected the two real rotations $R_{23}^{\theta}$ and $R_{13}^{\theta}$. Changing the parametrization:

$$
R_{13}^{\theta \dagger} R_{23}^{\theta \dagger} Q_{\text {rel }} R_{12}^{\varphi} R_{13}^{\varphi} \rightarrow Q_{\text {eff }} R_{12}^{\psi} R_{13}^{\psi} \tilde{R}_{23}^{\psi, \delta} Q_{\text {maj }}
$$


where $Q_{\text {eff }}=\operatorname{diag}\left(e^{i \beta_{1}}, e^{i \beta_{2}}, e^{-i \beta_{1}-i \beta_{2}}\right)$ are two new relative phases, $R_{12}^{\psi}$ and $R_{13}^{\psi}$ are real rotations by angles $\psi_{12}$ and $\psi_{13}, \tilde{R}_{23}^{\psi, \delta}$ is a complex rotation by angle $\psi_{23}$ and phase $\delta_{23}$, and $Q_{\text {maj }}$ contains two Majorana phases. The matrix on the l.h.s. is a det $=1$ unitary matrix, so it can be decomposed as in the r.h.s.. The new angles $\left(\psi_{12}, \psi_{13}, \psi_{23}\right)$ as well as the phases $\left(\delta_{23}, \beta_{1}, \beta_{2}\right)$ and the Majorana phases in $Q_{\text {maj }}$ are some complicated functions of the original angles $\left(\theta_{13}, \theta_{23}, \varphi_{12}, \varphi_{13}\right)$ and of the phases $\left(\alpha_{1}, \alpha_{2}\right)$, but they do not depend on $\Delta m_{31}^{2}, E_{\nu}$ (neutrino energy), $\varepsilon$, or $N_{e}(r)$. They are universal and independent of the details of the trajectory.

Now, obviously the Majorana phases $Q_{\text {maj }}$ disappear, as well as $\delta_{23}$ and $\psi_{23}$ (since they rotate the $(0,0)$ block in $\left.D_{\text {mat }}\right)$. Hence we are left with:

$$
H=R_{23}^{\theta} R_{13}^{\theta}\left[D_{\mathrm{vac}}+Q_{\mathrm{eff}} R_{12}^{\psi} R_{13}^{\psi} D_{\mathrm{mat}} R_{13}^{\psi \dagger} R_{12}^{\psi \dagger} Q_{\mathrm{eff}}^{\dagger}\right] R_{13}^{\theta \dagger} R_{23}^{\theta \dagger}
$$

Now we note that:

$$
D_{\mathrm{vac}}=Q_{\mathrm{eff}} R_{12}^{\psi} D_{\mathrm{vac}} R_{12}^{\psi \dagger} Q_{\mathrm{eff}}^{\dagger}
$$

since $R_{12}^{\psi}$ rotates the $(0,0)$ block in $D_{\text {vac }}$ and $Q_{\text {eff }}$ is diagonal. Therefore:

$$
H=U_{\text {eff }}\left[D_{\text {vac }}+R_{13}^{\psi} D_{\text {mat }} R_{13}^{\psi \dagger}\right] U_{\text {eff }}^{\dagger} \quad \text { where } \quad U_{\text {eff }} \equiv R_{23}^{\theta} R_{13}^{\theta} Q_{\text {eff }} R_{12}^{\psi}
$$

This lead to a scattering matrix:

$$
S=U_{\text {eff }} S_{\text {eff }} U_{\text {eff }}^{\dagger} \quad \text { with } \quad S_{\text {eff }}=\text { evolution-of }\left(D_{\text {vac }}+R_{13}^{\psi} D_{\text {mat }} R_{13}^{\psi \dagger}\right)
$$

It is immediate to see that the effective Hamiltonian $H_{\text {eff }}=D_{\text {vac }}+R_{13}^{\psi} D_{\text {mat }} R_{13}^{\psi \dagger}$ depends only on 3 parameters $\left(\Delta m_{31}^{2}, \varepsilon, \psi_{13}\right)$ and has the form (up to an overall multiple of the identity):

$$
H_{\mathrm{eff}}=\left(\begin{array}{ccc}
H_{D} & 0 & H_{N} \\
0 & H_{P} & 0 \\
H_{N} & 0 & -H_{D}
\end{array}\right) \quad \Longrightarrow \quad S_{\mathrm{eff}}=\left(\begin{array}{ccc}
S_{D} & 0 & -S_{N}^{*} \\
0 & S_{P} & 0 \\
S_{N} & 0 & S_{D}^{*}
\end{array}\right)
$$

hence it factorizes into a $2 \times 2$ block and a $1 \times 1$ block. The remaining 5 parameters $\theta_{13}$, $\theta_{23}, \beta_{1}, \beta_{2}, \psi_{12}$ are non-dynamical and can be reinserted later to form the $\chi^{2}$. Concretely, the neutrino probabilities can be written as the sum of 18 different terms:

$$
P_{\alpha \beta}=\sum_{n=1}^{18} C_{n}^{\alpha \beta} P_{n}^{\mathrm{eff}}
$$

where $C_{n}^{\alpha \beta}=C_{n}^{\alpha \beta}\left(\theta_{13}, \theta_{23}, \beta_{1}, \beta_{2}, \psi_{12}\right)$ and $P_{n}^{\text {eff }}=P_{n}^{\text {eff }}\left(\Delta m_{31}^{2}, \varepsilon, \psi_{13}\right)$ are real numbers re- 
lated to $U_{\text {eff }}$ and $S_{\text {eff }}$ by the following formulas:

$$
\begin{array}{rlrl}
C_{1}^{\alpha \beta} & =\left|W_{22}^{\alpha \beta}\right|^{2}+\left|W_{31}^{\alpha \beta}\right|^{2}+\left|W_{13}^{\alpha \beta}\right|^{2}, & & P_{1}^{\mathrm{eff}}=1, \\
C_{2}^{\alpha \beta} & =\left|W_{11}^{\alpha \beta}\right|^{2}+\left|W_{33}^{\alpha \beta}\right|^{2}-\left|W_{13}^{\alpha \beta}\right|^{2}-\left|W_{31}^{\alpha \beta}\right|^{2}, & P_{2}^{\mathrm{eff}} & =\left|S_{D}\right|^{2}, \\
C_{3}^{\alpha \beta}+i C_{4}^{\alpha \beta} & =W_{11}^{\alpha \beta^{*}} W_{31}^{\alpha \beta}-W_{13}^{\alpha \beta^{*}} W_{33}^{\alpha \beta}, & & P_{3}^{\mathrm{eff}}+i P_{4}^{\mathrm{eff}}=2 S_{D} S_{N}^{*}, \\
C_{5}^{\alpha \beta}+i C_{6}^{\alpha \beta} & =W_{11}^{\alpha \beta^{*}} W_{22}^{\alpha \beta}, & P_{5}^{\mathrm{eff}}+i P_{6}^{\mathrm{eff}}=2 S_{D} S_{P}^{*}, \\
C_{7}^{\alpha \beta}+i C_{8}^{\alpha \beta} & =W_{31}^{\alpha \beta^{*}} W_{22}^{\alpha \beta}, & P_{7}^{\mathrm{eff}}+i P_{8}^{\mathrm{eff}}=2 S_{N} S_{P}^{*}, \\
C_{9}^{\alpha \beta}+i C_{10}^{\alpha \beta} & =W_{11}^{\alpha \beta^{*}} W_{33}^{\alpha \beta}, & P_{9}^{\mathrm{eff}}+i P_{10}^{\mathrm{eff}}=2 S_{D} S_{D}, \\
C_{11}^{\alpha \beta}+i C_{12}^{\alpha \beta} & =-W_{31}^{\alpha \beta^{*}} W_{13}^{\alpha \beta}, & P_{11}^{\mathrm{eff}}+i P_{12}^{\mathrm{eff}}=2 S_{N} S_{N}, \\
C_{13}^{\alpha \beta}+i C_{14}^{\alpha \beta} & =W_{31}^{\alpha \beta^{*}} W_{33}^{\alpha \beta}-W_{11}^{\alpha \beta^{*}} W_{13}^{\alpha \beta}, & P_{13}^{\mathrm{eff}}+i P_{14}^{\mathrm{eff}}=2 S_{D} S_{N}, \\
C_{15}^{\alpha \beta}+i C_{16}^{\alpha \beta} & =W_{22}^{\alpha \beta^{*}} W_{33}^{\alpha \beta}, & P_{15}^{\mathrm{eff}}+i P_{16}^{\mathrm{eff}}=2 S_{D} S_{P}, \\
C_{17}^{\alpha \beta}+i C_{18}^{\alpha \beta} & =-W_{22}^{\alpha \beta^{*}} W_{13}^{\alpha \beta}, & P_{17}^{\mathrm{eff}}+i P_{18}^{\mathrm{eff}} & =2 S_{N} S_{P},
\end{array}
$$

with $W_{i j}^{\alpha \beta} \equiv U_{\alpha i}^{\text {eff }} U_{\beta j}^{\text {eff* }}$. For antineutrinos one has to flip the sign of the matter term in $H_{\text {eff }}$ and replace $U_{\text {eff }} \rightarrow U_{\text {eff }}^{*}$ (or, equivalently, $S_{\text {eff }} \rightarrow S_{\text {eff }}^{*}$ ) in eq. (A.11).

\section{B Symmetries and parameter ranges}

In order to describe the physical ranges for the parameters in the problem we study which kind of transformations leave the probabilities unaffected. Let's consider a problem described by an Hamiltonian $H$. The transformation $H \rightarrow-H^{*} \operatorname{implies} \exp (-i H L) \rightarrow$ $\exp \left(i H^{*} L\right)=\exp \left[(-i H L)^{*}\right]=[\exp (-i H L)]^{*}$, hence it leaves the probabilities unchanged. Such transformation is realized by:

(0) $\Delta m_{31}^{2} \rightarrow-\Delta m_{31}^{2} \wedge \varepsilon \rightarrow-\varepsilon \wedge \alpha_{1} \rightarrow-\alpha_{1} \wedge \alpha_{2} \rightarrow-\alpha_{2}$;

This symmetry implies that only the relative sign of $\Delta m_{31}^{2}$ and $\varepsilon$ matters, so that we can either assume $\varepsilon \geq 0$ and study both signs of $\Delta m_{31}^{2}$, or assume $\Delta m_{31}^{2} \geq 0$ and study both sign of $\varepsilon$. Furthermore, any rephasing $H \rightarrow Q H Q^{*}$ where $Q=\operatorname{diag}\left(e^{i a}, e^{i b}, e^{i c}\right)$ leads to a rephasing of the scattering matrix $\exp (-i H L) \rightarrow Q \exp (-i H L) Q^{*}$, which leaves the probabilities unaffected. Our Hamiltonian can be written as:

$$
H=U_{\mathrm{vac}} D_{\mathrm{vac}} U_{\mathrm{vac}}^{\dagger}+Q_{\mathrm{rel}} U_{\mathrm{mat}} D_{\mathrm{mat}} U_{\mathrm{mat}}^{\dagger} Q_{\mathrm{rel}}^{\dagger}
$$

where $U_{\text {vac }}=R_{23}\left(\theta_{23}\right) R_{13}\left(\theta_{13}\right)$ and $U_{\text {mat }}=R_{12}\left(\varphi_{12}\right) R_{13}\left(\varphi_{13}\right)$. A transformation $H \rightarrow$ $Q H Q^{*}$ is achieved if:

$$
U_{\mathrm{vac}} \rightarrow Q U_{\mathrm{vac}} Q^{\prime} \quad \text { and } \quad Q_{\mathrm{rel}} U_{\mathrm{mat}} \rightarrow Q Q_{\mathrm{rel}} U_{\mathrm{mat}} Q^{\prime \prime}
$$

where $Q, Q^{\prime}, Q^{\prime \prime}$ are all pure phase diagonal matrices. Hence, any transformation of the parameters which is equivalent to a specific choice of $Q, Q^{\prime}$ and $Q^{\prime \prime}$ will leave the probabilities unaffected. With this, we can generate a list of symmetries: 
(1) $\theta_{13} \rightarrow \pi+\theta_{13}$. This is equivalent to $Q^{\prime}=\operatorname{diag}(-1,+1,-1)$;

(2) $\theta_{13} \rightarrow-\theta_{13} \wedge \theta_{23} \rightarrow \pi+\theta_{23}$. This is equivalent to $Q^{\prime}=\operatorname{diag}(+1,-1,-1)$;

(3) $\varphi_{13} \rightarrow \pi+\varphi_{13}$. This is equivalent to $Q^{\prime \prime}=\operatorname{diag}(-1,+1,-1)$;

(4) $\varphi_{13} \rightarrow-\varphi_{13} \wedge \varphi_{12} \rightarrow \pi+\varphi_{12}$. This is equivalent to $Q^{\prime \prime}=\operatorname{diag}(-1,-1,+1)$;

(5) $\theta_{23} \rightarrow-\theta_{23} \wedge \varphi_{12} \rightarrow-\varphi_{12}$. This is equivalent to $Q=Q^{\prime}=Q^{\prime \prime}=\operatorname{diag}(+1,-1,+1)$;

(6) $\theta_{23} \rightarrow \pi-\theta_{23} \wedge \varphi_{12} \rightarrow \pi+\varphi_{12}$. This is equivalent to $Q=\operatorname{diag}(+1,+1,-1)$, $Q^{\prime}=\operatorname{diag}(+1,-1,+1), Q^{\prime \prime}=-I$

(7) $\varphi_{12} \rightarrow-\varphi_{12} \wedge \alpha_{1} \rightarrow \alpha_{1}+\pi / 3 \wedge \alpha_{2} \rightarrow \alpha_{2}-2 \pi / 3$. This is equivalent to $Q^{\prime \prime}=$ $e^{i \pi / 3} \operatorname{diag}(+1,-1,+1)$.

(8) $\varphi_{12} \rightarrow \pi+\varphi_{12} \wedge \alpha_{1} \rightarrow \alpha_{1}+\pi / 3 \wedge \alpha_{2} \rightarrow \alpha_{2}+\pi / 3$. This is equivalent to $Q^{\prime \prime}=e^{-2 i \pi / 3} I$.

With these symmetries we can reduce the range of the parameters $\left(\theta_{23}, \theta_{13}, \varphi_{12}, \varphi_{13}\right)$ from the most general $[0 \rightarrow 2 \pi]$ to something less:

- using (1) we can reduce $\theta_{13}$ to the range $[-\pi / 2 \rightarrow+\pi / 2]$;

- using $(2)$ we can reduce $\theta_{13}$ to the range $[0 \rightarrow \pi / 2]$;

- using (5) we can reduce $\theta_{23}$ to the range $[0 \rightarrow \pi]$;

- using (6) we can reduce $\theta_{23}$ to the range $[0 \rightarrow \pi / 2]$;

- using (3) we can reduce $\varphi_{13}$ to the range $[-\pi / 2 \rightarrow \pi / 2]$.

For the case of real NSI:

- $\operatorname{using}(4)$ we can reduce $\varphi_{12}$ to the range $[-\pi / 2 \rightarrow \pi / 2]$;

so that for $\alpha_{1}=\alpha_{2}=0$ we can reduce the parameter range for the angles to $0<\theta_{i j}<\pi / 2$ and $-\pi / 2<\varphi_{i j}<\pi / 2$. For the case of complex NSI:

- using (4) we can reduce $\varphi_{13}$ to the range $[0 \rightarrow \pi / 2]$;

- using (8) we can reduce $\varphi_{12}$ to the range $[-\pi / 2 \rightarrow \pi / 2]$;

- using (7) we can reduce $\varphi_{12}$ to the range $[0 \rightarrow \pi / 2]$;

so that in the general case of unconstrained $\alpha_{i}$ we can reduce the parameter range for the angles to $0<\theta_{i j}<\pi / 2$ and $0<\varphi_{i j}<\pi / 2$. 


\section{References}

[1] B. Pontecorvo, Neutrino experiments and the question of leptonic-charge conservation, Sov. Phys. JETP 26 (1968) 984 [SPIRES].

[2] V.N. Gribov and B. Pontecorvo, Neutrino astronomy and lepton charge, Phys. Lett. B 28 (1969) 493 [SPIRES].

[3] M.C. Gonzalez-Garcia and M. Maltoni, Phenomenology with Massive Neutrinos, Phys. Rept. 460 (2008) 1 [arXiv:0704.1800] [SPIRES].

[4] Z. Maki, M. Nakagawa and S. Sakata, Remarks on the unified model of elementary particles, Prog. Theor. Phys. 28 (1962) 870 [SPIRES].

[5] M. Kobayashi and T. Maskawa, CP Violation in the Renormalizable Theory of Weak Interaction, Prog. Theor. Phys. 49 (1973) 652 [SPIRES].

[6] S.M. Bilenky, J. Hosek and S.T. Petcov, On Oscillations of Neutrinos with Dirac and Majorana Masses, Phys. Lett. B 94 (1980) 495 [SPIRES].

[7] P. Langacker, S.T. Petcov, G. Steigman and S. Toshev, On the Mikheev-Smirnov-Wolfenstein (MSW) Mechanism of Amplification of Neutrino Oscillations in Matter, Nucl. Phys. B 282 (1987) 589 [SPIRES].

[8] T. Schwetz, M. Tortola and J.W.F. Valle, Global neutrino data and recent reactor fluxes: status of three-flavour oscillation parameters, arXiv:1103.0734 [SPIRES].

[9] M.C. Gonzalez-Garcia, M. Maltoni and J. Salvado, Updated global fit to three neutrino mixing: status of the hints of theta13 \& 0, JHEP 04 (2010) 056 [arXiv:1001.4524] [SPIRES].

[10] G.L. Fogli, E. Lisi, A. Marrone, A. Palazzo and A.M. Rotunno, Neutrino masses and mixing: 2008 status, Nucl. Phys. Proc. Suppl. 188 (2009) 27 [SPIRES].

[11] M. Maltoni and T. Schwetz, Three-flavour neutrino oscillation update and comments on possible hints for a non-zero $\theta_{13}$, PoS (idm2008) 072 [arXiv:0812.3161] [SPIRES].

[12] L. Wolfenstein, Neutrino oscillations in matter, Phys. Rev. D 17 (1978) 2369 [SPIRES].

[13] S.P. Mikheev and A.Y. Smirnov, Resonance enhancement of oscillations in matter and solar neutrino spectroscopy, Sov. J. Nucl. Phys. 42 (1985) 913 [SPIRES].

[14] G.L. Fogli, E. Lisi, A. Palazzo and A.M. Rotunno, Solar neutrino oscillations and indications of matter effects in the sun, Phys. Rev. D 67 (2003) 073001 [hep-ph/0211414] [SPIRES].

[15] E. Roulet, MSW effect with flavor changing neutrino interactions, Phys. Rev. D 44 (1991) 935 [SPIRES].

[16] M.M. Guzzo, A. Masiero and S.T. Petcov, On the MSW effect with massless neutrinos and no mixing in the vacuum, Phys. Lett. B 260 (1991) 154 [SPIRES].

[17] V.D. Barger, R.J.N. Phillips and K. Whisnant, Solar neutrino solutions with matter enhanced flavor changing neutral current scattering, Phys. Rev. D 44 (1991) 1629 [SPIRES].

[18] G.L. Fogli and E. Lisi, Solar Neutrino data, solar model uncertainties and solar matter enhanced neutrino oscillations, Astropart. Phys. 2 (1994) 91 [SPIRES].

[19] S. Bergmann, The solar neutrino problem in the presence of flavor changing neutrino interactions, Nucl. Phys. B 515 (1998) 363 [hep-ph/9707398] [SPIRES]. 
[20] S. Bergmann, M.M. Guzzo, P.C. de Holanda, P.I. Krastev and H. Nunokawa, Status of the solution to the solar neutrino problem based on nonstandard neutrino interactions, Phys. Rev. D 62 (2000) 073001 [hep-ph/0004049] [SPIRES].

[21] M.M. Guzzo, H. Nunokawa, P.C. de Holanda and O.L.G. Peres, On the massless 'just-so' solution to the solar neutrino problem, Phys. Rev. D 64 (2001) 097301 [hep-ph/0012089] [SPIRES].

[22] A. Friedland, C. Lunardini and C. Pena-Garay, Solar neutrinos as probes of neutrino matter interactions, Phys. Lett. B 594 (2004) 347 [hep-ph/0402266] [SPIRES].

[23] F.J. Escrihuela, O.G. Miranda, M.A. Tortola and J.W.F. Valle, Constraining nonstandard neutrino-quark interactions with solar, reactor and accelerator data, Phys. Rev. D 80 (2009) 105009 [arXiv: 0907.2630] [SPIRES].

[24] A. Bolanos, O.G. Miranda, A. Palazzo, M.A. Tortola and J.W.F. Valle, Probing non-standard neutrino-electron interactions with solar and reactor neutrinos, Phys. Rev. D 79 (2009) 113012 [arXiv:0812.4417] [SPIRES].

[25] H. Minakata and C. Pena-Garay, Solar Neutrino Observables Sensitive to Matter Effects, arXiv: 1009.4869 [SPIRES].

[26] A. Palazzo, Hint of non-standard dynamics in solar neutrino conversion, arXiv:1101.3875 [SPIRES].

[27] Y. Grossman, Nonstandard neutrino interactions and neutrino oscillation experiments, Phys. Lett. B 359 (1995) 141 [hep-ph/9507344] [SPIRES].

[28] M.C. Gonzalez-Garcia, Y. Grossman, A. Gusso and Y. Nir, New CP-violation in neutrino oscillations, Phys. Rev. D 64 (2001) 096006 [hep-ph/0105159] [SPIRES].

[29] A.M. Gago, M.M. Guzzo, H. Nunokawa, W.J.C. Teves and R. Zukanovich Funchal, Probing flavor changing neutrino interactions using neutrino beams from a muon storage ring, Phys. Rev. D 64 (2001) 073003 [hep-ph/0105196] [SPIRES].

[30] N. Fornengo, M. Maltoni, R. Tomas and J.W.F. Valle, Probing neutrino non-standard interactions with atmospheric neutrino data, Phys. Rev. D 65 (2002) 013010 [hep-ph/0108043] [SPIRES].

[31] P. Huber and J.W.F. Valle, Non-standard interactions: Atmospheric versus neutrino factory experiments, Phys. Lett. B 523 (2001) 151 [hep-ph/0108193] [SPIRES].

[32] T. Ota, J. Sato and N.-a. Yamashita, Oscillation enhanced search for new interaction with neutrinos, Phys. Rev. D 65 (2002) 093015 [hep-ph/0112329] [SPIRES].

[33] P. Huber, T. Schwetz and J.W.F. Valle, Confusing non-standard neutrino interactions with oscillations at a neutrino factory, Phys. Rev. D 66 (2002) 013006 [hep-ph/0202048] [SPIRES].

[34] M. Campanelli and A. Romanino, Effects of new physics in neutrino oscillations in matter, Phys. Rev. D 66 (2002) 113001 [hep-ph/0207350] [SPIRES].

[35] T. Ota and J. Sato, Can ICARUS and OPERA give information on a new physics?, Phys. Lett. B 545 (2002) 367 [hep-ph/0202145] [SPIRES].

[36] M.C. Gonzalez-Garcia and M. Maltoni, Atmospheric neutrino oscillations and new physics, Phys. Rev. D 70 (2004) 033010 [hep-ph/0404085] [SPIRES]. 
[37] A. Friedland, C. Lunardini and M. Maltoni, Atmospheric neutrinos as probes of neutrino matter interactions, Phys. Rev. D 70 (2004) 111301 [hep-ph/0408264] [SPIRES].

[38] A. Friedland and C. Lunardini, A test of tau neutrino interactions with atmospheric neutrinos and K2K, Phys. Rev. D 72 (2005) 053009 [hep-ph/0506143] [SPIRES].

[39] M. Blennow, T. Ohlsson and W. Winter, Non-standard Hamiltonian effects on neutrino oscillations, Eur. Phys. J. C 49 (2007) 1023 [hep-ph/0508175] [SPIRES].

[40] N. Kitazawa, H. Sugiyama and O. Yasuda, Will MINOS see new physics?, hep-ph/0606013 [SPIRES].

[41] A. Friedland and C. Lunardini, Two modes of searching for new neutrino interactions at MINOS, Phys. Rev. D 74 (2006) 033012 [hep-ph/0606101] [SPIRES].

[42] M. Blennow, T. Ohlsson and J. Skrotzki, Effects of non-standard interactions in the MINOS experiment, Phys. Lett. B 660 (2008) 522 [hep-ph/0702059] [SPIRES].

[43] J. Kopp, M. Lindner and T. Ota, Discovery reach for non-standard interactions in a neutrino factory, Phys. Rev. D 76 (2007) 013001 [hep-ph/0702269] [SPIRES].

[44] J. Kopp, M. Lindner, T. Ota and J. Sato, Non-standard neutrino interactions in reactor and superbeam experiments, Phys. Rev. D 77 (2008) 013007 [arXiv:0708.0152] [SPIRES].

[45] N.C. Ribeiro, H. Minakata, H. Nunokawa, S. Uchinami and R. Zukanovich-Funchal, Probing Non-Standard Neutrino Interactions with Neutrino Factories, JHEP 12 (2007) 002 [arXiv:0709.1980] [SPIRES].

[46] ISS Physics Working Group collaboration, A. Bandyopadhyay et al., Physics at a future Neutrino Factory and super-beam facility, Rept. Prog. Phys. 72 (2009) 106201 [arXiv: 0710.4947] [SPIRES].

[47] N.C. Ribeiro et al., Probing Nonstandard Neutrino Physics by Two Identical Detectors with Different Baselines, Phys. Rev. D 77 (2008) 073007 [arXiv:0712.4314] [SPIRES].

[48] A. Esteban-Pretel, J.W.F. Valle and P. Huber, Can OPERA help in constraining neutrino non-standard interactions?, Phys. Lett. B 668 (2008) 197 [arXiv:0803.1790] [SPIRES].

[49] M. Blennow, D. Meloni, T. Ohlsson, F. Terranova and M. Westerberg, Non-standard interactions using the OPERA experiment, Eur. Phys. J. C 56 (2008) 529 [arXiv:0804.2744] [SPIRES].

[50] J. Kopp, T. Ota and W. Winter, Neutrino factory optimization for non-standard interactions, Phys. Rev. D 78 (2008) 053007 [arXiv:0804.2261] [SPIRES].

[51] T. Ohlsson and H. Zhang, Non-Standard Interaction Effects at Reactor Neutrino Experiments, Phys. Lett. B 671 (2009) 99 [arXiv:0809.4835] [SPIRES].

[52] A. Palazzo and J.W.F. Valle, Confusing non-zero $\theta_{13}$ with non-standard interactions in the solar neutrino sector, Phys. Rev. D 80 (2009) 091301 [arXiv:0909.1535] [SPIRES].

[53] M. Blennow and T. Ohlsson, Approximative two-flavor framework for neutrino oscillations with non-standard interactions, Phys. Rev. D 78 (2008) 093002 [arXiv:0805.2301] [SPIRES].

[54] S. Davidson, C. Pena-Garay, N. Rius and A. Santamaria, Present and future bounds on non-standard neutrino interactions, JHEP 03 (2003) 011 [hep-ph/0302093] [SPIRES].

[55] C. Biggio, M. Blennow and E. Fernandez-Martinez, Loop bounds on non-standard neutrino interactions, JHEP 03 (2009) 139 [arXiv:0902.0607] [SPIRES]. 
[56] CHOOZ collaboration, M. Apollonio et al., Limits on Neutrino Oscillations from the CHOOZ Experiment, Phys. Lett. B 466 (1999) 415 [hep-ex/9907037] [SPIRES].

[57] Kamiokande collaboration, R. Wendell et al., Atmospheric neutrino oscillation analysis with sub-leading effects in Super-Kamiokande I, II and III, Phys. Rev. D 81 (2010) 092004 [arXiv: 1002.3471] [SPIRES].

[58] The MINOS collaboration, P. Adamson et al., Measurement of the neutrino mass splitting and flavor mixing by MINOS, Phys. Rev. Lett. 106 (2011) 181801 [arXiv:1103.0340] [SPIRES].

[59] P. Valhe, New results from MINOS, talk given at the XXIV International Conference on Neutrino Physics and Astrophysics, Athens Greece, June 14-19 2010.

[60] The MINOS collaboration, P. Adamson et al., New constraints on muon-neutrino to electron-neutrino transitions in MINOS, Phys. Rev. D 82 (2010) 051102 [arXiv:1006.0996] [SPIRES].

[61] J. Kopp, P.A.N. Machado and S.J. Parke, Interpretation of MINOS data in terms of non-standard neutrino interactions, Phys. Rev. D 82 (2010) 113002 [arXiv:1009.0014] [SPIRES].

[62] W.A. Mann, D. Cherdack, W. Musial and T. Kafka, Apparent multiple Delta $m_{32}^{2}$ in muon anti-neutrino and muon neutrino survival oscillations from non-standard interaction matter effect, Phys. Rev. D 82 (2010) 113010 [arXiv: 1006.5720] [SPIRES].

[63] E. Akhmedov and T. Schwetz, MiniBooNE and LSND data: non-standard neutrino interactions in a (3+1) scheme versus (3+2) oscillations, JHEP 10 (2010) 115 [arXiv: 1007.4171] [SPIRES].

[64] G.L. Fogli, E. Lisi, A. Marrone, A. Palazzo and A.M. Rotunno, Hints of $\theta_{13}>0$ from global neutrino data analysis, Phys. Rev. Lett. 101 (2008) 141801 [arXiv:0806.2649] [SPIRES]. 